\section{The 4th Annual Meeting of the American Society for Nanomedicine}

Howard E. Gendelman, Lajos P. Balogh, Raj Bawa, Michelle Bradbury, Esther H. Chang, Wah Chiu, Omid Farokhzad, Marianna Foldvari, Gregory Lanza and Kuan Wang

\section{The Board of Directors of the American Society for Nanomedicine}

Correspondence:

Howard E. Gendelman

985880 Nebraska Medical Center

Omaha, NE 68198-5880

Phone: 4025598920

Fax: 4025593744

Email: hegendel@unmc.edu

\section{Abstract}

The 4th Conference of the American Society for Nanomedicine is being held March 28-30, 2014 at the Universities at Shady Grove, Rockville, Maryland. The meeting's theme is on defining the role of nanomedicines for nervous system diagnostics and disease but balanced by broad and timely topics for nanotechnology. Nanoneuromedicine, as defined by the development of small drug formulations for the diagnosis and treatment of degenerative, inflammatory, infectious, vascular, addictive, behavioral and metabolic disorders of the nervous system, will provide a focus for each of the scientific sessions. This research is interdisciplinary and it's in its infancy. The hurdles that preclude translation from bench to bedside would include its delivery across the blood brain barrier, limiting nervous system toxicities, and improving drug targeting to diseased brain subregions. These all pose challenges. Multidisciplinary works in neuroscience (neurobiology, neurochemistry, neurophysiology, and neuroinflammation), bioimaging, and polymer chemistry to facilitate outcomes for formulation manufacture will be vigorously discussed. How drugs reach sites of action need include neural cell specific subcellular compartments. The ASNM meeting will showcase nanoneuromedicine research from leading investigators of divergent scientific backgrounds who define this new field. It will also serve as an incubator for developing investigators and broad new field discoveries. Welcome to the conference and enjoy!

Keywords: Nanomedicine, Blood Brain Barrier; Product translation, Neuroimaging, Diagnostics, Neurotherapeutics, Drug targeting, Nanotoxicity
The board of directors of the American Society for Nanomedicine (ASNM) is pleased to welcome you to our March 28-30, 2014 conference held at The Universities of Shady Grove, Rockville, Maryland. The meeting will foster the vision and goals of the society to promote basic, clinical and population-based research in nanotechnology, engineering, biochemistry, molecular biology, and medicine. A rich program is offered that will educate all our delegates including physicians, scientists, engineers, molecular biologists, statisticians, and many others interested in this emerging and exciting field. Opportunities will abound during the next several days for vigorous exchange through the meeting and in subsequent publications offered through Nanomedicine: Nanotechnology, Biology and Medicine and the Journal of Neuroimmune Pharmacology. Our short and long-term goals are to encourage primary and secondary preventive measures using state of the art nanomedicine technologies to reduce the incidences of a broad range of diseases. We trust the meeting will serve to speed research efforts aimed at a better quality and duration of life through the types of drug discovery and improved diagnostics being discussed at the conference. A significant focus of these efforts is aimed towards promoting and facilitating the formal training of our physicians, scientists, engineers, molecular biologists, statisticians and other members and allied healthcare providers in the nanotechnology, nanobiology and nanomedicine fields.

The 2014 theme of the conference - nanoneuroscience focuses on drug delivery to the nervous system for substance abuse, degenerative medicine, mental health, infectious diseases, and cancer. This two and one-half day meeting features scientific sessions on CNS drug and formulation design, targeted delivery and toxicology, diagnostics at the nanoneural interface, neuronanotherapeutics, neuroregenerative medicine, bench to bedside translations and applications for nanomedicines, legal, developmental, scientific and regulatory obstacles for product translation, and how nanomedicines can be used and harnessed for HIV eradication. We anticipate that each of these cross-disciplinary sessions can readily provide significant opportunities for early career investigators to meet and interact with scientific and clinical leaders in the field as well as provide opportunities for future collaboration amongst investigators. The interaction with funding agencies, pharmaceutical companies, and other industry leaders will also be available through the conference.

Andre $\mathrm{Nel}, \mathrm{MbChB}, \mathrm{PhD}$, will open the meeting with his keynote address "Nanomedicine: The Key to an Engineered Approach to Medicine and Biology." Dr. Nel is Chief and Founder of NanoMedicine in the Department of Medicine, University of California, Los Angeles (UCLA) with broad expertise in the research of novel nanotherapeutic platforms 
for strong clinical translation. This presentation will set the stage for the meeting, in toto.

Gregory Lanza, MD, PhD, FACC, Washington University, St. Louis and Kuan Wang, PhD, Academia Sinica will chair the session on "CNS drug and formulation design, targeted delivery and toxicology." Speakers include Alexander Kabanov, PhD, Dr SCI, University of North Carolina; Esther Chang, PhD, Georgetown University Medical Center; Bruce Hinds, PhD, University of Kentucky; Steven Rannard, PhD, University of Liverpool; Jinjun Shi, PhD, Harvard Medical School; and Wei-Yi Ong, National University of Singapore. This session will discuss brain tumor targeting, transdermal addiction therapy, and development of novel sustained release nanotherapies that include siRNA and particle biodistribution.

Raj Bawa, PhD, Rensselaer Polytechnic Institute and Andrew Salperstein, PhD, GSK Pharmaceuticals will lead the session on "Scientific, regulatory and developmental obstacles for product translation." Presenters will include Scott Livingston, BA, Livingston Securities; Joseph A. Frank, MD, MS, Clinical Center and National Institute of Biomedical Imaging and Bioengineering, National Institutes of Health; Susan Rosenbaum, JD, Lauren Sciences LLC; Shivani Ghaisas, Medical College of Wisconsin; and Thomas Ernst, $\mathrm{PhD}$, University of Hawaii Medical Center. This session will serve as a bridge between the legal and scientific aspects of new technologies being developed in diagnostics and in using nanotechnologies for tissue regeneration and disease amelioration. The focus will be innovation and translation.

Steve Jacobson, PhD, National Institute of Neurological Disease and Stroke (NINDS) and Wah Chiu, Baylor College will chair and present in the session on "Diagnostics at the nanoneural interface." This session also includes Elad Alon, PhD, University of California, Berkley; Dipanjan Pan, PhD, University of Illinois at Urbana-Champaign; Yuri Lyubchenko, PhD, DrSc, University of Nebraska Medical Center(UNMC); Steve Jacobson, PhD, NINDS; Linda Chang, MD of the University of Hawaii Medical Center; and Wah Cui, $\mathrm{PhD}$, Baylor College of Medicine. The focus will be on novel technologies to improve diagnostics and disease monitoring for a range of neurologic and degenerative disorders.

Marianna Foldvari, PhD, University of Waterloo and Avindar Nath, MD, NINDS, will chair the "Nanotherapeutics" session. Speakers include Omid Farokhzad, MD, Brigham and Women's Hospital; Gregory Lanza, MD Washington University School of Medicine; Jagat Kanwar, PhD, Deakin University; Christine Pham, MD, Washington University, St. Louis; Vladimir Muzykantov MD, PhD, University of Pennsylvania; and Xiaoyuan Chen, $\mathrm{PhD}$, National Institute of Biomedical Imaging and Bioengineering. This session will cover novel nanotherapeutics from the laboratory bench to the patient bedside for a range of neurologic and peripheral disorders.

Lajos Balogh, PhD, Nanomedicine \& Nanotechnology, Boston, and Omid Farokhzad, MD, Brigham and Women's Hospital will chair the session on "Neuroregenerative Medicine.” Presentations from; KiBum Lee, PhD, Rutgers University; Kam Leong, PhD, Duke University; Matthew Kelso, PharmD, PhD, UNMC; Michal Toborek MD, PhD, University of Miami; and Marianna Foldvari, $\mathrm{PhD}$ will cover the promise and perils for neuroregenerative medicine.

Jonathan Pollock, PhD, National Institute on Drug Abuse and Elena Batrakova, PhD, University of North Carolina College of Pharmacy will lead the session on "Bench to Bedside: Applications for Nanomedicines." This session brings together Elena Batrakova, PhD; George Perry, PhD, University of Texas, San Antonio; Surya Mallapragada, PhD, Iowa State University; Michelle Bradbury, MD, PhD, Memorial Sloan-Kettering Cancer Center; Gabriel Lopez-Berestein, MD, University of Texas MD Anderson Cancer Center; and Ravi Bellamkonda PhD, Georgia Institute of Technology and Emory University. This session will focus squarely on the newest avenues for nanomedicine research in combating brain disease. It will cover critical research aspects from the cells to humans.

A special session is devoted to early career investigators. Michelle Bradbury, MD, PhD, Memorial Sloan-Kettering Cancer Center and Sulie Chang, PhD, Seton Hall University will lead this session. Lajos Balogh, PhD, Editor-in-Chief, Nanomedicine NBM, will provide an editor's view of nanomedicine. Through an NIH R13 conference grant, the society was able to provide 12 travel awards (oral and poster). Additional presentations from the next generation of leaders on critical advances in the field include Eric C. Peterson, PhD, University of Arkansas for Medical Sciences; David Leong, National University of Singapore; Julijana Ivanisevic, PhD, The Scripps Research Institute; Janet Hsu, BS, University of Maryland; and Xi Zhu, BS, Harvard Medical School.

Jeymohan Joseph, PhD, NIMH and May Wong, PhD, NINDS will lead the session on "Nanomedicine for HIV eradication in the brain and other reservoirs." Speakers include Andrew Owen, PhD, University of Liverpool; Jerome Zack, PhD, UCLA; Stanley Schwartz and Jessica Reynolds, State University of NY at Buffalo; Howard E. Gendelman, MD, and Serguei Vinogradov, PhD, DrSc, UNMC. This session will discuss in some depth how the latest avenues of nanomedicine research have and will strongly impact the field of HIV/AIDS for treatment and eradication.

A vigorous poster session will engage most of the topic sessions with a strong focus on early career professionals. Posters will be shown throughout the conference and showcased Friday night, March 28th. 
The meeting will wrap up with a round table discussion moderated by Esther Chang, PhD and Howard E. Gendelman, MD. The session will give all participants an opportunity to discuss the future direction, innovation, and needs in the field.

Once again welcome and enjoy. We hope you find the platform and discussions of great interest and the collaborations and discussions exciting.
The conference was supported by the National Institutes of Health, National Institute of Neurological Disorders and Stroke R13 Scientific Conference grant 1R13NS083315-01; Seton Hall University; UNMC; Carol Swarts, MD, Emerging Neuroscience Research Laboratory, UNMC; ViiV Healthcare Company; Springer publisher, Journal of Neuroimmune Pharmacology. 


\title{
Fourth Annual Conference of the American Society for Nanomedicine
}

\author{
Universities at Shady Grove, Rockville Maryland \\ March 28-30, 2014
}

\section{Conference Organizers}

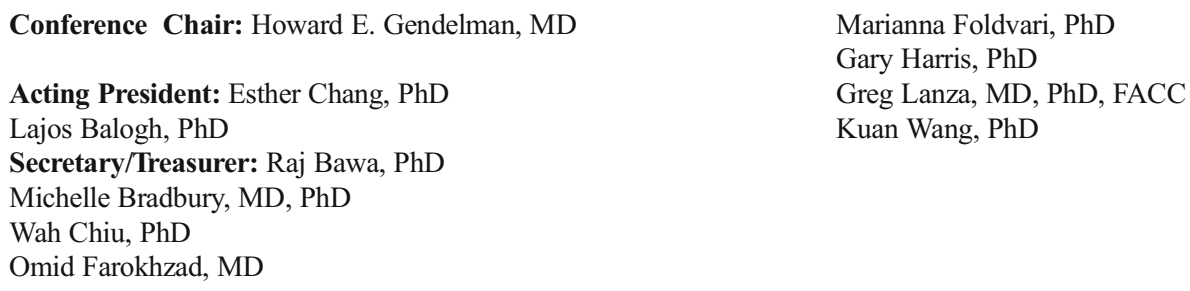

Thursday, March 27, 2014

6:00 p.m.

American Society for Nanomedicine Leadership Dinner (by invitation)

Friday, March 28, 2014

7:00 a.m. -5 p.m.

8:00 a.m

$8: 15$ a.m

9:00 a.m.

9:05-9:25 a.m.

9:25-9:45 a.m.

9:45-10:05 a.m

10:05-10:25 a.m.

10:25-10:45 a.m.

10:45-11:05 a.m.

11:05-11:20 a.m

11:20 a.m.

11:25-11:35 a.m.
Registration Desk Open

Welcome

Esther Chang, PhD

Introduction to Lecture

Howard E. Gendelman, MD

Keynote Address: Andre Nel, MBChB, PhD, University of California, Los Angeles

"Nanomedicine: The Key to an Engineered Approach to Medicine and Biology"

Session 1: CNS drug and formulation design, targeted delivery and toxicology

Co-Chairs: Greg Lanza, MD, PhD, FACC, Washington University, St. Louis

Kuan Wang, PhD, Academia Sinica

Alexander Kabanov, PhD, Dr SCI, University of North Carolina

"A Very High Capacity Polymeric Micelle Drug Delivery System"

Esther Chang, PhD, Georgetown University Medical Center

"Brain Tumor-Targeting Nanomedicine Sensitizes Glioblastoma to Chemotherapy and Improves Survival"

Bruce Hinds, PhD, University of Kentucky

"Gated Carbon Nanotube Membranes for Transdermal Addiction Therapy"

Steven Rannard, PhD, University of Liverpool

"Emerging Platform Technologies for Nanotherapies"

Jinjun Shi, PhD, Harvard Medical School

"Self-Assembled Lipid-Polymer Hybrid Nanoparticles for Sustained siRNA Delivery"

Wei-Yi Ong, PhD, BDS, National University of Singapore

"Effect of Inhalation Exposure on Biodistribution of Nanoparticles, Including the Brain"

Break

Session 2. Scientific, regulatory and developmental obstacles for product translations

Co-Chairs: Raj Bawa, PhD, Rensselaer Polytechnic Institute: Bawa Biotech LLC

Andrew Spaltenstein, PhD, GSK Pharmaceuticals

Scott Livingston, BA, Livingston Securities

"The JOBS Act, Crowdfunding, and the Return of the Small IPO: Changing the Way Innovation is financed on Wall Street" 
11:35-11:55 a.m.

11:55-12:15 p.m.

12:15-12:35 p.m.

12:35-12:50 p.m.

12:50-1:45 p.m.

1:45 p.m.

1:50-2:10 p.m.

2:10-2:30 p.m.

2:30-2:50 p.m.

2:50-3:10 p.m.

3:10-3:30 p.m.

3:30-3:50 p.m.

3:50-4:05 p.m.

4:20 p.m.

4:25-4:45 p.m.

4:45-5:05 p.m.

5:05-5:25 p.m.

5:25-5:45 p.m.

5:45-6:05 p.m.

6:05-6:25 p.m.

6:30-7:30 p.m.
Joseph A. Frank, MD, MS, Clinical Center and National Institute of Biomedical Imaging and Bioengineering, National Institutes of Health

"Stem Cell Imaging Bench to Bedside"

Susan Rosenbaum, JD, Lauren Sciences LLC

"Product Development and Translations: From Cancer to HIV and NeuroAIDS"

Thomas Ernst, PhD, University of Hawaii Medical Center

"Innovation and Translation into the Marketplace"

Shivani Ghaisas, Medical College of Wisconsin (Early Career Investigator)

"Development of a Nanoparticle-Based Mitochondrial-Targeted Antioxidant Therapy Platform for

Treatment of Chronic Traumatic Encephalopathy"

\section{Box Lunch (available to purchase) and Poster Viewing}

Session 3: Diagnostics at the Nanoneural Interface

Co-Chairs: Steve Jacobson, PhD, National Institute of Neurological Disease and Stroke

Wah Chiu, PhD, Baylor College of Medicine

Elad Alon, PhD, University of California, Berkeley

"Neural Dust An Ultrasonic, Low-Power Solution for Brain-Machine Interfaces"

Dipanjan Pan, PhD, University of Illinois at Urbana-Champaign

"Quantitative Imaging of Coronary Ruptured Plaque with Energy-Resolved Spectral Computed

Tomography and K-edge Metal Nanocolloids without Calcium Interference"

Yuri Lyubchenko PhD, DrSc, University of Nebraska Medical Center

"Amyloid Hypothesis for AD Insight from Single Molecule Experiments and Computational Analyses"

Steve Jacobson, PhD, National Institute of Neurological Disorders and Stroke

"Nanomedicine in Demyelinating Disease: Application to Diagnosis and Therapy in Neuroinflammatory Disorders"

Linda Chang, MD, University of Hawaii Medical Center

"MR Studies to Assess HIV-Associated Brain Injury Current and Future Perspectives"

Wah Chiu, PhD, Baylor College of Medicine

"Electron Cryo-tomography of Cells and Protein Aggregates"

\section{Break}

Session 4: Nanotherapeutics

Chairs: Marianna Foldvari, PhD, University of Waterloo

Avindra Nath, MD, National Institute of Neurological Disorders and Stroke

Omid Farokhzad, MD, Brigham and Women's Hospital

"Targeted Polymeric Nanoparticles from Discovery to Clinical Trials"

Greg Lanza, MD, PhD, FACC, Washington University School of Medicine

"Scanning Probe Technologies for Carotid Microthombus Diagnoses Using Spectral CT"

Jagat R. Kanwar, PhD, Deakin University

"Nanoformulated Cell Penetrating Survivin Mutant and Lactoferrin Induced Differentiation and PGlycoprotein Mediated Anti-Epileptic Drug Resistance Reversal"

Christine T.N. Pham, MD, Washington University, St. Louis

"Characterization of Nanoparticle Complement Interaction"

Vladimir Muzykantov MD, PhD, University of Pennsylvania

"Endothelial Nanomedicine"

Xiaoyuan Chen, PhD, National Institute of Biomedical Imaging and Bioengineering

${ }^{، 64} \mathrm{Cu}$-Doped Nanoparticles for In Vivo Tumor Imaging and Therapy"

\section{Poster Session I and Social Hour}

Saturday, March 29, 2014

7:30 a.m. -6 p.m. Registration Desk Open 
8:00 a.m.

8:05-8:25 a.m.

8:25-8:45 a.m.

8:45-9:05 a.m.

9:05-9:25 a.m.

9:25-9:45 a.m.

9:45-10:00 a.m.

10:00 a.m

10:05-10:25 a.m.

10:25-10:45 a.m.

10:45-11:05 a.m.

11:05-11:25 a.m.

11:25-11:45 a.m.

11:45-12:05 p.m.

12:05-1:15 p.m.

1:15 p.m.

1:20-1:40 p.m.

1:40-2:00 p.m.

2:00-2:20 p.m.

2:20-2:40 p.m.

3:00-3:15 p.m.

3:15-3:30 p.m.
Session 5: Neuroregenerative Medicine: Promise and Perils

Co-Chairs: Lajos Balogh, PhD, AA Nanomedicine \& Nanotechnology

Omid Farokhzad, MD, Brigham and Women's Hospital

KiBum Lee, PhD, Rutgers University

"Nanotechnology Approaches For Controlling Stem Cell Fate"

Kam Leong, PhD, Duke University

"Cell-Topography Interactions and Neuronal Differentiation"

Matthew Kelso, PharmD, PhD, University of Nebraska Medical Center

"Neuroprotective Regulatory T Cells as Vehicles for Nanoformulated Growth Factor Delivery

Following Traumatic Brain Injury"

Michal Toborek MD, PhD, University of Miami

"Perspective for Nanotoxicology"

Marianna Foldvari, PhD, University of Waterloo

"Gene Therapy Approaches to Regenerating the Optic Nerve"

Break

Session 6: Bench to Bedside: Applications for nanomedicines

Co-Chairs: Jonathan Pollock, PhD, National Institute on Drug Abuse

Elena Batrakova, PhD, University of North Carolina, College of Pharmacy

Elena Batrakova, PhD, University of North Carolina, College of Pharmacy

"Carriers that Break Barriers"

George Perry, PhD, University of Texas, San Antonio

"Mitochondria at the Origin of Oxidative Stress"

Surya Mallapragada, PhD, Iowa State University,

"Nanomedicines for Neuroprotection and Neuroregeneration"

Michelle Bradbury, MD, PhD, Memorial Sloan-Kettering Cancer Center

"Ultrasmall Hybrid Inorganic Nanoparticle Probes for Brain Tumor Imaging"

Gabriel Lopez-Berestein, MD, University of Texas MD Anderson Cancer Center

"Targeting Neurons with Chitoisan Nanoparticles"

Ravi Bellamkonda PhD, Georgia Institute of Technology and Emory University

"Nanofiber and Nanocarrier Mediated Modulation of Cell Migration for Nerve Repair and Brain Tumor Therapy"

Box Lunch (available to purchase) and Poster Viewing

Session 7: Early Career Investigators

Co-Chairs: Michelle Bradbury, MD, PhD, Memorial Sloan-Kettering Cancer Center Sulie Chang, PhD, Seton Hall University

Lajos Balogh, PhD, Editor-in-Chief, Nanomedicine NBM (Elsevier)

"Nanomedicine: An Editor's View"

Eric C. Peterson, PhD, University of Arkansas for Medical Sciences (Early Career Investigator)

"Nanomedicine and Toxicology"

David Leong, PhD, National University of Singapore (Early Career Investigator)

"Nanoparticle Induced Endothelial Leakiness Mechanism and Implications to Nanomedicine"

Julijana Ivanisevic, PhD, The Scripps Research Institute (Early Career Investigator)

"Metabolic Phenotyping to Functionally Characterize Brain Regions"

Janet Hsu, BS, University of Maryland (Early Career Investigator)

"Transport of ICAM-1-Targeted Nanoparticles Across Endothelial-Subendothelial Layers and Uptake by Neurons"

Xi Zhu, BS, Harvard Medical School (Early Career Investigator)

"Self-Assembled Lipid-Polymer Hybrid Nanoparticles for the Sustained Delivery of Small Interfering RNA" 
3:45-4:00 p.m. Group Photo

4:00-4:15 p.m. $\quad$ Break

4:15-4:45 p.m.

4:45-6:15 p.m.

7-9:30 p.m.
ASNM Business Meeting (members and non members welcome)

Special Session: Poster Viewing (Early Career Investigator) and Early Career Investigator Education Session

Chairs: Michelle Bradbury and Howard E. Gendelman

Meet the Professor/Young Investigator Dinner (by invitation)

Location: TBA

Sunday, March 30, 2014

8:30 a.m. - 11 a.m.

9:00 a.m.

9:05-9:25 a.m.

9:25-9:45 a.m.

9:45-10:05 a.m.

10:05-10:25 a.m.

10:25-10:45 a.m.

10:45-11:05 a.m.

11:10 -11:30 a.m.

11:30 a.m.
Registration Desk Open

Session 8: Nanomedicine for HIV Eradication in Brain and Other Reservoirs

Co-Chairs: Jeymohan Joseph, PhD, National Institute of Mental Health

May Wong, PhD, National Institute of Neurological Disease and Stroke

Andrew Owen, PhD, University of Liverpool

"Sustained Release Formulations for HIV Therapy"

Jerome Zack, PhD, University of California, Los Angeles

"A Nanoparticle Approach to Targeting Latent HIV Reservoir"

Stanley Schwartz, MD, PhD, State University of New York at Buffalo

"Nanotherapies: Current and Future Challenges"

Howard E. Gendelman, MD, University of Nebraska Medical Center

"Nanoformulated Antiretroviral Therapy and the CNS"

Serguei Vinogradov, PhD, DrSc, University of Nebraska Medical Center

"Application of Targeted Nano-NRTI Cocktails against HIV Infection in the Brain"

Jessica Reynolds, PhD, State University of NY at Buffalo (Early Career Investigator)

"Pharmacokinetic and Immune-Dynamics of Multimodular particles for HIV and/or TB"

Questions and Closing Remarks

Co-Chairs: Howard E. Gendelman and Esther Chang

Meeting Adjourned 


\section{Keynote Address}

\section{NANOMEDICINE: THE KEY TO AN ENGINEERED APPROACH TO MEDICINE AND BIOLOGY}

Nel A. David Geffen School of Medicine, University of California, Los Angeles 90095.

Since much of structure and function of biology is executed at nanoscale level, this provides us with an exciting new opportunity to use the unique properties of engineered nanomaterials for interrogation of disease, diagnosis, treatment, and imaging at levels of sophistication not previously accomplished. Nanomedicine can be defined as the design and synthesis of biologically interactive nanoscale systems that enable the biomedical community to make advances in the prevention, diagnosis, and treatment of disease, maintenance and improvement of human health, and providing chronic and acute pain relief by leveraging significant advantages nanosystems hold over traditional methods for sensing, imaging, reconstruction, delivery and interaction with biological systems. This vision is being implemented by our UCLA Nanomedicine Division and a multidisciplinary working group that performs basic, translational and clinical-based research using the unique properties of a range of engineered nanomaterials to understand the nano/bio interface as a launching pad for developing new diagnostic and therapeutic modalities. Fabrication of a host of nanostructures in a collaborative setting in the California NanoSystems Institute (CNSI) is being coupled with advanced chemical manipulation to develop diagnostics and therapeutics for infectious disease, cancer, immunological disease. This includes a multifunctional mesoporous nanoparticle imaging and delivery system for therapeutics that are based on the pathophysiology of disease and clinical-defined problems. We also use high throughput screening and modeling approaches to assist nanomaterial design and safety assessment. We envisage the use of nanotechnology and materials science to develop a series of engineered approaches by physician/ scientists teams that tackle clinical defined disease problems.

\section{SESSION 1: CNS DRUG AND FORMULATION DESIGN, TARGETED DELIVERYAND TOXICOLOGY}

\section{A VERY HIGH CAPACITY POLYMERIC MICELLE DRUG DELIVERY SYSTEM}

Kabanov A ${ }^{1}$, Jordan $\mathrm{R}^{2}$, Luxenhofer $\mathrm{R}^{3} .{ }^{1}$ University of North Carolina at Chapel Hill, Chapel Hill, NC 27599-7362;

${ }^{2}$ Technische Universität Dresden, ${ }^{3}$ University Würzburg.

Polymeric micelles proposed as carriers for drug delivery in the end of 80 -ies by Kabanov's and Kataoka's groups have now reached a clinical stage with one polymer micelle product approved and number of others undergoing clinical trials. One "value proposition" of polymeric micelles is their ability to incorporate water-insoluble drugs. However, only few drugs and polymeric micelle systems displayed loading capacities of even $10-20 \%$ (wt. drug/wt. drug and polymer) with most others having lower capacities. We discovered that polymeric micelles of poly(2-methyl-2-oxazoline) (PMeOx) and poly(2butyl-2-oxazoline) (PBuOx) triblock copolymer PMeOx$\mathrm{PBuOx}-\mathrm{PMeOx}$ have an unprecedented high loading capacity of nearly $50 \%$ for paclitaxel (PTX), docetaxel (DTX) as well as other drugs and multi-drug combinations. Such loading capacity is due to a unique multi-domain structure of the PBuOx hydrophobic core, which can accommodate both hydrophobic and polar groups of many drug molecules. As a result the amount of the polymer used is greatly decreased (e.g. nearly 50 times compared to Chremophor EL in Taxol) and the maximal tolerated dose (MTD) increased (e.g. in a mouse 7 times vs. Taxol). Due to greater exposure at the increased and safe dose the drug more efficiently kills the tumor cells resulting in more efficient therapy. Multiple drugs and drug combinations can be solubilized providing for a versatile drug delivery platform for 1) rescuing "undruggable" molecules; 2) reformulating existing drugs; and 3) creating multi-drug combinations that affect synergistic pathways and more efficiently cure the disease.

Supported by: Cancer Nanotechnology Platform Partnership grant (U01 CA116591).

\section{BRAIN TUMOR-TARGETING NANOMEDICINE SENSITIZES GLIOBLASTOMATO CHEMOTHERAPY AND IMPROVES SURVIVAL}

Chang $\mathrm{EH}^{1,2}$, Kim $\mathrm{SS}^{1}$, Rait $\mathrm{A}^{1}$, Kim $\mathrm{E}^{2}$, Pirollo $\mathrm{KF}^{1}$. ${ }^{1}$ Department of Oncology, Lombardi Comprehensive Cancer Center, Georgetown University, Washington, DC 20057; ${ }^{2}$ SynerGene Therapeutics, Inc., Potomac, MD 20854.

Temozolomide (TMZ)-resistance in glioblastoma multiforme (GBM) has been linked to upregulation of O6-methylguanineDNA methyltransferase (MGMT). However, therapy for GBM is often limited by lack of efficient delivery across the blood brain barrier (BBB). We have developed a systemically administered, tumor-targeting nanodelivery system comprising a liposome directed to the tumor by a single-chain antibody fragment targeted to the transferrin receptor. This selfassembled nanocomplex ( $\mathrm{scL}$ ), shown to encapsulate various payloads including nucleic acid-based therapeutics, diagnostic imaging probes, small molecules, and chemotherapeutic agents, exhibits exquisite tumor specificity in numerous preclinical studies. The nanocomplex carrying the wtp53 gene (SGT-53) is currently being evaluated in a number of clinical trials where it has been shown to be well tolerated and has demonstrated anti-cancer activity. Here, we show that SGT-53 crosses the BBB and efficiently targets GBM, as well as cancer stem cells (CSCs). Wtp53 was previously 
shown to downmodulate MGMT. Thus, we also evaluated the effect of scL-p53 on MGMT expression in a mouse model of GBM. Systemic delivery of SGT-53 downmodulated MGMT and induced apoptosis in intracranial GBM xenografts. The combination of SGT-53 and TMZ increased the anti-tumor efficacy of TMZ with enhanced survival benefit in a mouse model of highly TMZ-resistant GBM. SGT-53 sensitized both CSCs and bulk tumor cells to TMZ, increasing apoptosis. These results suggest that combining SGT-53 with standard TMZ treatment could be a more effective therapy for GBM.

\section{GATED CARBON NANOTUBE MEMBRANES FOR TRANSDERMAL ADDICTION THERAPY}

Hinds B. Chemical and Materials Engineering, University of KY, Lexington, KY 40506.

Addiction treatment is one of the most difficult health care challenges due to the mixture of complex changing neurochemical pathways and psychological behavior. Generally the most effective treatments require psychological monitoring/ counseling and adaption of therapeutic techniques. For large population addiction, such as nicotine, it is cost and time prohibitive to have face to face meetings. A promising system is where a dosing regiment (within a doctors' prescription limit) can be remotely programmed to account for daily environmental factors, patient input, and counselor feedback from phone interviews or internet-based surveys. Needed for this system is an ultra-low power, compact, and programmable delivery device not currently available with electroporation or mechanical pumps.

Carbon nanotubes (CNTs) are electrically conductive and support dramatic internal flow rates [Nature 2005]. These properties are nearly ideal for introducing efficient electrophoretic and electro-osmotic flow [Nat. Nanotech 2011] to be used as the basis of a programmed transdermal delivery device. These CNT membranes are 100 fold more energy efficient than conventional nanoporous materials allowing watch battery operation for 1 week. An in-vitro cell, composed of a reference electrode, reservoir solution, CNT membrane electrode, gel contact and human skin sample were assembled in a Franz cell. Therapeutically useful fluxes for Nicotine treatment were controllably switched between, with 0.56 and 2.0 micromole/cm2-hr at $0 \mathrm{mV}$ and $-600 \mathrm{mV}$ respectively [PNAS 2010]. Prototype device studies on hairless guinea pigs showed active switching [J. Pharm. Sci. 2012] with further refinement of time dependent dosing using microdialysis membrane assays in vitro. Wireless Bluetooth programmed control with basic survey input was also demonstrated allowing for the combination of psychological and physiological approaches in addiction treatments.

Supported by: NIH NIDA R01DA018822

\section{EMERGING PLATFORM TECHNOLOGIES FOR NANOTHERAPIES}

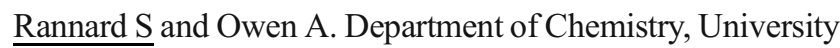
of Liverpool, Liverpool, UK L69 7ZD.

Nanomedicines have been very successful in the development of new therapies from existing active pharmaceutical ingredients (APIs) and have heavily targeted direct intravenous delivery or oral administration. Diseases/conditions ranging from cancer to organ rejection are treated daily by nanoparticle technologies. The most successful platform to date is the nanomilling of poorly soluble APIs to form solid drug nanoparticles, which are orally dosed although extended research programmes and clinical studies are investigating the benefits of milled SDNs for sustained delivery from depot injections. Material chemistry has a strong role to play in the provision of options for platform approaches to nanotherapy development. Nanomilling is not applicable to all APIs and the rapid production of libraries of nanomilled SDNs is time consuming. A recent platform has been developed that utilizes novel freeze-drying approaches and as little as $1 \mathrm{mg}$ of API per library member. Diversity of SDN chemistry, size and surface charge may be readily studied across $100 \mathrm{~s}$ of candidates. New polymeric nanocarriers are also being developed that have the benefits of ideal dendrimers but the flexibility and scalability of conventional polymeric materials. This overview will examine the advances in freeze drying technologies and new polymeric nanocarrier systems and relate their value to the treatment of HIV.

\section{SELF-ASSEMBLED LIPID-POLYMER HYBRID NANOPARTICLES FOR SUSTAINED SIRNA DELIVERY}

Shi, J. Brigham and Women's Hospital, Harvard Medical Center, Boston, MA 02115.

RNA interference (RNAi), which can selectively knockdown target genes, has shown great potential in the treatment of various diseases including cancer. Thus far, numerous nanoparticle (NP) platforms, such as lipoplexes and polyplexes, have been developed to facilitate the safe and effective delivery of small interfering RNA (siRNA), which represents a major hurdle for the clinical applications of RNAi. Nevertheless, these NP systems lack the sustained siRNA release property, and thus can only induce transient gene silencing due to the short lifetime of siRNA. Therefore, it is expected that the development of controlled-release siRNA NPs will lead to sustained gene silencing and more effective cancer treatment. 
Herein, we present a robust NP platform for safe and effective siRNA delivery in a sustained manner, which can be developed through the self-assembly of biodegradable and biocompatible polymers and lipids. The lipid-polymer hybrid NPs show excellent knockdown efficacy at low doses of siRNA. More importantly, these NPs can control the temporal release of siRNA, with the half-release time of $\sim 9$ days, for sustained silencing of target gene expression. For example, results demonstrate that the expression of PHB1 can be effectively inhibited for over 2 weeks after short-term transfection with the NPs. The NP-mediated sustained silencing of PHB1 in turn generates more effective tumor cell growth inhibition in vitro and in vivo than the lipofectamine-siRNA complexes. Furthermore, this NP platform has been applied to deliver siRNAs against drug resistance and chemotherapeutic drugs for synergistic cancer treatment. We expect that the lipid-polymer hybrid NP platform with the property of sustained siRNA release could be of interest in both fundamental biological studies and clinical applications.

\section{EFFECT OF INHALATION EXPOSURE ON BIODISTRIBUTION OF NANOPARTICLES, INCLUDING THE BRAIN}

Ong WY. Department of Anatomy, Yong Loo Lin School of Medicine, National University of Singapore 119597.

Background: Gold nanoparticles (AuNPs) are finding increased use in therapeutics and imaging. This study was carried out to determine the biodistribution and gene effects of AuNPs after inhalation exposure. Methods Male Wistar rats were exposed for 15-days to airborne agglomerates of AuNPs of similar size distribution and number concentration $(1 \times 10(6)$ particles $/ \mathrm{cm}(3))$, but two different primary diameters of $7 \mathrm{~nm}$ or $20 \mathrm{~nm}$, and whole body biodistribution of Au determined by ICP-MS. Possible gene expression changes were also determined in the ling, kidney and hippocampus by microarrays. Results: After inhalation exposure, the highest mass concentration of agglomerates containing 7-nm AuNPs were deposited in the lungs, followed by brain regions including the olfactory bulb, hippocampus, striatum, frontal cortex, entorhinal cortex, septum, cerebellum; aorta, esophagus, and kidney. Eight organs/tissues especially the brain retained greater mass concentration of $\mathrm{Au}$ after inhalation exposure to agglomerates of 7-nm than 20-nm AuNPs. Macrophage mediated escalation followed by fecal excretion is the major pathway of clearing inhaled AuNPs in the lungs. Microarray analyses of the lung showed mostly downregulated genes related to muscle and secretion. The hippocampus showed mostly downregulated genes, related to the cytoskeleton and neurite outgrowth. In comparison, after intravenous injection, $\mathrm{Au}$ was rapidly and consistently accumulated in liver (49.4+/$50.4-72.2+/-40.5 \mathrm{ng} / \mathrm{g})$ and spleen $(8.4+/-5.0-9.5+/-6.4 \mathrm{ng} / \mathrm{g})$. Conclusion: The above results demonstrate differential biodistribution of AuNPs in the body after inhalation exposure or intravenous injection, and gene expression changes in target organs.

\section{SESSION 2. SCIENTIFIC, REGULATORY AND DEVELOPMENTAL OBSTACLES FOR PRODUCT TRANSLATIONS} RETURN OF THE SMALL IPO: CHANGING THE WAY INNOVATION IS FINANCED ON WALL STREET Livingston S. Livingston Securities, New York, NY 10022.

The biotech revolution was built by 2 million share IPOs at $\$ 8$ The internet was built by 3 million share IPOs at $\$ 12$.

Emerging technology companies in the late 80 s and 90 s had access to IPOs to help them raise capital to scale up their breakthrough technologies. In addition, having a public company and a ticker symbol offered many other advantages to help companies grow.

A number of companies succeeded wildly, many companies failed, and many other companies were acquired and merged into bigger companies. Along the way the biotech industry created millions of jobs, offered new treatments for diseases, and created over a trillion dollars of wealth.

After the dot com bust a number of rules were put into place that changed this environment. While many of the changes were needed, unforeseen impacts also arose and the small IPO effectively disappeared in the US.

The breakthrough technologies of the 21st century (nanotechnology, personalized medicine, regenerative medicine, etc) have had to grow up in a much different environment than the biotechs of the early 90s. Many companies have complained that a lack of access to scale up capital has been their main challenge.

In April of 2012 the Congress passed and President Obama signed the JOBS Act into law. One result of the JOBS Act has been a significant increase in the IPO markets, and in particular the smaller IPO, which has had a major impact on financing disruptive nano-bio companies.

Livingston Securities participated in 31 equity capital markets transactions (IPOs and secondaries) in 2013, vs 25 in 2012 and 5 in 2011 . We have been very active in helping emerging nano-bio companies raise capital in the public and private markets over the last few years, and are well versed and positioned to help companies understand how these changes can impact their capital raising efforts, as well as to help institutional and individual investors make smart investment decisions in the new post-JOBS Act environment. 


\section{STEM CELL IMAGING BENCH TO BEDSIDE}

Frank JA. Clinical Center and National Institute of Biomedical Imaging and Bioengineering, Bethesda, MD 20892.

Over the past decade there has been increasing interest in the potential use cellular therapies to treat diseases. Labeling transplanted cells to be identified on MRI offers the ability to track the migration of transplants cells into tissues, as part of treatment strategy is information that is potentially unavailable by other methods. Various techniques have been developed to label cells with MRI contrast agents. Both experimental and clinically used paramagnetic (gadolinium, manganese) and superparamagnetic iron oxide (SPION) nanoparticles have been used to label stem cells, progenitor cells, immune cells and cancer cell lines for cellular MRI studies. Although there are a variety of agents that have been used to tag cells for in vivo imaging studies, this presentation will focus on the use of MRI contrast agents to track cells. I will also discuss the use of ferumoxytol, a recently approved ultrasmall SPION for iron deficiency anemia that can be used to magnetically label cells ex vivo for cellular MRI. In order to label cell In particular I will address specific questions including: 1) is there a need to track cells by MRI; 2) what agents can be used to label cells with the goal to translate from the bench to bedside; 3) how long can labeled cells be tracked by MRI and what is needed to noninvasively determine cell function, survival or differentiation; 4) what happens to the label in tissues; and 5) Will magnetic cell labeling be used in the clinical by 2020? Part of the presentation will discuss the challenges in taking magnetic cell labeling with SPION to the clinic trials, the possible preclinical data for regulatory approval, along with the potential of where magnetic labeling of cell tagging may be useful in cell therapy.

\section{PRODUCT DEVELOPMENT AND TRAN SLATIONS: FROM CANCER TO HIVAND NEUROAIDS}

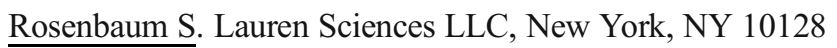

The process of commercializing an invention, taking a discovery and making it into a medicine, getting it from the Ph.D.s and giving it to the patients, is referred to as going from "bench to bedside". But, what exactly does this mean? And, what does it entail?

Product development, or translations from research into development to clinic, will be explored by way of example. The case study will be of Lauren Sciences LLC, a start up biotech with a breakthrough technology in nanomedicine, and a current focus on neurodegenerative diseases. Lauren Sciences has a patent estate of 10 patent families, and 15 papers published in peer-reviewed scientific journals.

Lauren Sciences is developing transformative V-SmartTM therapeutics based on its groundbreaking innovation - the VSmartTM targeted drug delivery system. V-SmartTM is a novel nanovesicle platform technology invented at Ben Gurion University. V-SmartTM nanovesicles can cross the $\mathrm{BBB}$, target inside the brain, selectively release at target sites and be administered systemically.

Lauren Sciences pipeline includes transformative V-SmartTM therapeutics for: Parkinson's, AIDS, Alzheimer's, ALS, Brain Cancer and LSDs. Lauren Sciences recently demonstrated successful delivery, by i.v. administration, of: (1) GDNFloaded V-SmartTM nanovesicles targeted to dopaminergic neurons in the brain for the treatment of Parkinson's (supported by The Michael J. Fox Foundation), and (2) Tenofovirloaded V-SmartTM nanovesicles to the brain for the treatment of neuro-HIV (supported by The Campbell Foundation).

The challenges of product translations, whether scientific, regulatory or developmental, require an integrated approach and a strategic plan. All applications of a product, whether for cancer or AIDS, will satisfy unmet medical needs. It takes people and project synergies to make it happen. The new model used by Lauren Sciences cuts to the chase.

\section{INNOVATION AND TRANSLATION INTO THE MARKETPLACE}

Ernst T. Department of Medicine, University of Hawaii, Honolulu, HI 96813.

The ultimate goal of biomedical research, including nanomedicine, is to improve human health. This requires that basic research findings and innovations are eventually translated into the commercial realm. The two major pathways towards commercialization of intellectual property (IP) are 1) creation of a startup company, and 2) licensing of the IP to an existing business. The choice between these two paths is partially driven by the potential market size of the final product, with option 1 (startups) being more favorable for larger markets (typically $\$ 1$ billion or greater). Compared to licensing, creation of a startup company to commercialize IP has several potential advantages, due to a tight focus of the new company on turning the IP into a marketable product. Difficulties and disadvantages associated with commercialization via a startup company include 1) potentially high risk of failure due to the narrow focus, typically on a single product, 2) the need to raise capital funds, 3) potentially long time frame for entry into the market (years), and 4) the inventors may lose control over research and product development. However, the laser-like focus of typical startup companies also maximizes chances for success in commercializing the IP. Also, since startup companies take on early risk, successful commercialization can result in large gains to the inventors and the Universities where the research was conducted. Ultimately, inventors may draw a substantial reward from seeing their invention being turned into a real-life product that will benefit patients. 
012. DEVELOPMENT OF A NANOPARTICLE-BASED MITOCHONDRIAL-TARGETED ANTIOXIDANT THERAPY PLATFORM FOR TREATMENT OF CHRONIC TRAUMATIC ENCEPHALOPATHY

Ghaisas $\mathrm{S}^{1}$, Brenza $\mathrm{T}^{2}$, Zenitsky $\mathrm{G}^{1}$, Vela-Ramirez $\mathrm{J}^{2}$, Anantharam $\mathrm{V}^{1}$, Joseph $\mathrm{J}^{3}$, Kalyanaraman $\mathrm{B}^{3}$, Kanthasamy $\mathrm{A}^{1}$, Mallapragada $\mathrm{S}^{2}$, Narasimhan $\mathrm{B}^{2}$ and Kanthasamy $\mathrm{AG}^{1}$. ${ }^{1}$ Departments of Biomedical Sciences, and ${ }^{2}$ Chemical and Biological Engineering, Iowa State University, Ames, IA 50011; ${ }^{3}$ Department of Biophysics, Medical College of Wisconsin, Milwaukee, WI 53226.

Effective neuroprotective strategies to treat chronic traumatic encephalopathy (CTE) induced neurodegenerative diseases, such as Parkinson's (PD) and Alzheimer's, are urgently needed. Recently, we showed that mitochondria-targeted antioxidants can be effective against dopaminergic neuronal degeneration in cell culture and animal models of PD. The goal of this study is to evaluate the efficacy of a novel mitochondrial-targeted antioxidant nanoformulation, MitoApocynin Encapsulated Nanoparticles (MEN), in cell culture and animal models of CTE. After first encapsulating mitoapocynin with poly (sebacic acid) into MEN, we then determined that 30-100 $\mu \mathrm{g}$ of MEN was optimal for in vitro studies as this dose range did not cause any significant toxicity over a $24 \mathrm{~h}$ period in neuronal cultures. Additionally, $30 \mu \mathrm{g}$ of $1 \% \mathrm{MEN}$ protected against glutamateinduced excitotoxicity in primary cortical neurons based on Sytox and MTT assays. To develop a robust in vitro CTE model for testing MEN's efficacy, we used the Cell Injury Controller II system to deliver strain-induced trauma to in vitro cell cultures and brain slices. Stretch injury using primary cortical neurons consistently showed elevated caspase- 3 enzymatic activity vs sham-blast, indicating the model's viability for determining apoptotic cell death after blast induced stretch injury. In developing animal studies of CTE, we examined behavioral and neurochemical changes in a mouse blast injury model. C57BL/6 black mice exposed to a single-blast or repeated blasts of 20 psi showed mild behavioral and neurochemical deficits on day one post-blast but recovered by day seven post-blast. We also measured neuroinflammatory markers including TNF $\alpha$, IL-1 $\beta$, IL-6, iNOS and MMP9 mRNA. Current studies are investigating MEN in brain slice and mouse models of CTE. Together, these findings indicate that nanoparticle-encapsulated mitochondria-targeted antioxidants may represent a novel nanomedicine-based treatment strategy for CTE.

\section{SESSION 3: DIAGNOSTICS AT THE NANONEURAL INTERFACE}

013. NEURAL DUST AN ULTRASONIC, LOW-POWER SOLUTION FOR BRAIN-MACHINE INTERFACES

Alon E, Seo DJ, Carmena J, Rabaey J, Maharbiz M. University of California, Berkeley, Berkeley, CA 94720.
A major hurdle in brain-machine interfaces (BMI) is the lack of an implantable neural interface system that remains viable for a lifetime. I will discuss Neural Dust, a concept developed with DJ Seo, Michel Maharbiz, Jose Carmena, and Jan Rabaey (http://arxiv.org/abs/1307.2196), which explores the fundamental system design trade-offs and ultimate size, power, and bandwidth scaling limits of neural recording systems built from low-power silicon (CMOS) circuitry coupled with ultrasonic power delivery and backscatter communication. In particular, we propose an ultra-miniature as well as extremely compliant system that enables massive scaling in the number of neural recordings from the brain while providing a path towards truly chronic BMI. These goals are achieved via two fundamental technology innovations: 1) large numbers of 10 100 micron scale, free-floating, independent sensor nodes, or neural dust, that detect and report local extracellular electrophysiological data, and 2) a sub-cranial interrogator that establishes power and communication links with the neural dust.

\section{QUANTITATIVE IMAGING OF CORONARY RUPTURED PLAQUE WITH ENERGY-RESOLVED SPECTRAL COMPUTED TOMOGRAPHY AND K- EDGE METAL NANOCOLLOIDS WITHOUT CALCIUM INTERFERENCE}

Schirra $\mathrm{CO}^{1,2}$, Senpan $\mathrm{A}^{1}$, Roessl $\mathrm{E}^{2}$,Thran $\mathrm{A}^{2}$, Scott $\mathrm{MJ}^{1}$, Gaffney $\mathrm{PJ}^{1}$, Wickline $\mathrm{SA}^{1}$, Proksa ${ }^{2}$, Lanza GM ${ }^{1}$, Pan $\mathrm{D}^{2,3}$. ${ }^{1}$ Philips Research, 22335 Hamburg, Germany; ${ }^{2}$ CTRAIN, Division of Cardiology, Washington University School of Medicine, St. Louis, MO 63110; ${ }^{3}$ Department of Bioengineering, University of Illinois at Urbana-Champaign, Beckman Institute, Carle Foundation Hospital, Urbana, IL 61820.

Emerging development of SPECTRAL CT and the opportunity for k-edge-based molecular imaging capability is expected to eliminate coronary calcium interference and offer ruptured coronary plaque detection. Spectral CT generates images based on unique spectral footprints of specific metals based on K-edge differences in the X-ray energy band, which will also permit detection and quantification of ruptured plaque with fibrin-specific K-edge contrast agents. The goal of these experiments was to introduce new class of molecular imaging agents, based on bismuth $(\mathrm{Bi})$ and ytterbium $(\mathrm{Yb})$, to detect microthombus in ruptured atherosclerotic plaque in combination with an advanced statistical image reconstruction method for improved signal-to-noise ratio (SNR). We hypothesized that rapid Spectral CT imaging in combination with targeted contrast agents that carry K-edge material will provide a revolutionary improvement in the recognition, quantification, and stratification of coronary plaque instability and guide medical decisions for early percutaneous intervention versus aggressive medical therapy. Metal nanobeacons (MNB) were designed and synthesized by concentrating a high payload of metals within a vascularly constrained 
$(\sim 200 \mathrm{~nm})$ particle. MNBs targeted to fibrin acellular in vitro phantoms and human carotid specimens ex vivo revealed high contrast signal and intravascular constraint. Noninvasive in vivo detection of thrombus with anti-fibrin MNBs was demonstrated in circulating blood using a balloon overstretch injury-induced thrombus formed within the iliac artery of an atherosclerotic rabbit. For this study, anti-fibrin $\mathrm{mAb}$ was coupled directly to MNB (Bi) particles. Bioelimination study revealed that $95 \%$ of the metal was cleared in 7 days and $>96 \%$ at 14 days in rodents. This study reports one of the first examples of targeted Spectral CT molecular imaging demonstrating the potential to detect ruptured plaque and eliminate calcium interference with $\mathrm{CT}$ vascular imaging.

\section{AMYLOID HYPOTHESIS FOR AD INSIGHT} FROM SINGLE MOLECULE EXPERIMENTS AND COMPUTATIONAL ANALYSES

Lyubchenko Y. Department of Pharmaceutical Sciences, University of Nebraska Medical Center, Omaha, NE 68198.

The current model for the development of Alzheimer's (AD), Parkinson's, Huntington's, prion, and other neurodegenerative diseases involves protein misfolding as the early step followed by spontaneous aggregation, with specific proteins identified as the primary initiators for disease development. Therefore, elucidating the properties of the disease-prone misfolded states, understanding the mechanism of their formation, and identification of their most toxic forms will open prospects for the development of early diagnostics and specific therapeutics for these diseases. We have developed single molecule AFM force spectroscopy (SMFS) experimental approach enabling us to probe interprotein interactions and to identify those interactions that correspond to misfolded protein states. Using SMFS, we have discovered that misfolded dimers are very stable. $A \beta 42$ and $A ß 40$ are the two primary alloforms of the amyloid $\beta$-protein, and we applied SMFS approaches to characterize the effects of $\mathrm{C}$-terminal substitutions on the structure of transiently formed dimers. We discovered a dramatic difference in the folding patterns of $A B 42$ and Aß40 monomers within dimers. Although the sequence difference between the two peptides is at the C-termini, the N-terminal segment plays a key role in the peptide folding in the dimers. To address the question on the mechanism of the misfolded dimers formation we applied Molecular Dynamics simulations. When two monomers approach, their structure changes dramatically. The arrangement of monomers in an antiparallel orientation leads to the cooperative formation of a $\beta$-sheet conformer. The amyloid misfolding depends on the environmental conditions and AFM is capable of characterizing these effects. Partially supported by: NIH 5R01 GM096039-04 and NSF EPS-1004094
016. NANOMEDICINE IN DEMYELINATING DISEASE: APPLICATION TO DIAGNOSIS AND THERAPY IN NEUROINFLAMMATORY DISORDERS

Jacobson S. National Institute of Neurological Disorders and Stroke, Bethesda, MD 20892.

Multiple sclerosis (MS) is the most prevalent inflammatory, demyelinating disease of the CNS. Lesions within myelinated regions of the CNS, mediated by infiltrating $\mathrm{T}$ cells are a hallmark of the disease and lead to progressive neurologic dysfunction. While the etiological agent(s) of MS remains unknown, the heterogeneous disease course suggests a host of complex interactions between immunologic, genetic and environmental factors. Similar to other inflammatory diseases, the pathogenesis of a rare, human retrovirus (HTLV-I) associated neurologic disorder has been suggested to involve interplay between host genes and antigen-specific immune responses. HTLV-I-associated myelopathy/tropical spastic paraparesis (HAM/TSP) is clinically similar to the chronic progressive form of MS. It is likely that a more complete understanding of the pathogenesis of a neurologic disease with a known viral etiology (HAM/TSP) will aid in defining mechanisms of pathogenesis of MS, a disease of unknown etiology in which viruses have been suspected to play a role. Clinical, pathological, and immunological events in HAM/ TSP have been studied. While progress has been made in characterizing the role of cellular immune responses to HTLV-I in HAM/TSP, there remains a significant gap in knowledge about the inflammatory pathways that are altered, and how HTLV-I contributes to this dysregulation. Our ability to translate knowledge gained from these basic virologic/ immunologic studies into clinically effective treatments represents a unique opportunity to better understand how virallytriggered immune dysregulation leads to neurologic disease. As the past decade has seen rapid advances in the integration of nanotechnology and medicine, there is a compelling need for the application of 'nanomedicine' to the diagnosis and treatment of inflammatory disorders of the nervous system. Examples will be highlighted from our studies on HAM/TSP and MS that will focus on the detection of virus and virusspecific immune responses in the CNS. In addition, imaging of these diseases have led to a better understanding of the pathogenesis of these disorders. The potential for nanoparticles to ferry drugs and contrast agents across the BBB will likely facilitate more efficacious and precise drug delivery and disease assessment by MRI.

Supported by: Intramural Program of the NINDS, NIH

\section{MR STUDIES TO ASSESS HIV-ASSOCIATED BRAIN INJURY CURRENT AND FUTURE PERSPECTIVES}

Chang L and Ernst T. John A. Burns School of Medicine, University of Hawaii at Manoa, Honolulu, Hawaii 96821. 
Despite effective viral suppression with antiretroviral therapy, $30-50 \%$ of HIV patients continue to show HIVassociated neurocognitive disorders (HAND) and evidence of ongoing neuroinflammation. HAND is often difficult to diagnose in the clinical settings, and will become more prevalent due to the aging population. Better clinical diagnostic tools are needed. A variety of MR techniques can evaluate the brain changes. Quantitative MRI consistently found atrophy in subcortical brain regions in HIV patients with or without HAND. Proton MR spectroscopy demonstrated ongoing neuroinflammation, and functional MRI studies showed lower cognitive reserve in HIV patients. These brain measures are typically related to the severity of cognitive deficits. Furthermore, particular genetic variants (e.g., APOE or COMT) in HIVinfected individuals are associated with greater brain atrophy or greater age-dependent decline in brain function. Hence, genetic markers and neuroimaging measures may provide useful endophenotypes to identify HIV patients at risk for HAND or predict treatment outcomes.

Novel MR contrast agents such as superparamagnetic iron oxide (SPIO), comprising nano-sized iron oxide crystals coated with dextran or carboxydextran (60-180 nm), can evaluate macrophage activities associated with neuroinflammation (e.g., in patients with ischemic strokes, multiple sclerosis and carotid plaques). Therefore, these agents may be useful for assessing neuroinflammation associated with HIV. Furthermore, the distribution of nanomedicine (e.g., SMART) or cell-based therapies in target organs, including the brain, can be assessed quantitatively and qualitatively when combined with SPIO agents. These nano-sized contrast agents are also ideal for translational studies for medication development. These emerging applications of SPIO and other potential new approaches to assess HIV-associated brain injury will be discussed.

Supported by: NIH (NIDA, NINDS, NIMH, NIMHD)

\section{ELECTRON CRYO-TOMOGRAPHY OF CELLS AND PROTEIN AGGREGATES}

Chiu W. National Center for Macromolecular Imaging, Baylor College of Medicine, Houston, TX 77030.

Electron cryo-tomography (Cryo-ET) has become a critical structural tool that brings structural insights that cannot be obtained by crystallography or single particle electron cryomicroscopy. The advance in instrumentation has made recording tilt series from ice-embedded specimens without chemical fixative and metal stain relatively routine using a transmission electron microscope equipped with a cryo-specimen holder kept at liquid nitrogen temperature. The reconstructed 3-D tomogram reveals vast amount of sub-cellular features in the crowded cells or biochemically prepared protein aggregates. The technical challenges are to annotate the observed structural features from the 3-D tomograms and to quantify them. Two examples will be presented to demonstrate how cryo-ET followed by extensive data processing can yield mechanistic insights in a biochemical phenomenon of inhibiting protein aggregation by a chaperonin and the spatial organization of the subcellular components in the rat hippocampal neurite grown on an electron microscope grid.

\section{SESSION 4: NANOTHERAPEUTICS}

\section{TARGETED POLYMERIC NANOPARTICLES FROM DISCOVERY TO CLINICAL TRIALS}

Farokhzad O. Brigham \& Women's Hospital/Harvard Medical School, Boston MA 02115.

A variety of organic and inorganic materials have been utilized to generate nanoparticles for drug delivery applications. The goal of this talk is to review our efforts in the design and optimization of polymeric nanoparticles for medical applications, which formed the foundation for the clinical translation of the first-in-human targeted and controlled-release nanoparticles, BIND-014 and SEL-068.

\section{SCANNING PROBE TECHNOLOGIES FOR CAROTID MICROTHOMBUS DIAGNOSES USING SPECTRAL CT}

Schmieder A, Pan D, Schirra C, Cui G, Senpan A, Zhang H, Yang X, Scott M, Allen J, Wickline S, Lanza GM. Washington University Medical School and Philips Healthcare, St. Louis, MO 63108.

Since the early work of Benson and Constantinides the acute formation of thrombus following atherosclerotic plaque rupture has been well recognized as the etiology of unstable angina, myocardial infarction, transient ischemic attacks and stroke. The most common source of thromboembolism are rupturing vulnerable plaques that reside in vessels with only 40 to $60 \%$ residual stenosis. Recent guideline changes have encouraged that asymptomatic patients with $>60 \%$ ipsilateral stenosis and $<3 \%$ surgical risk undergo carotid endarterectomy (CEA) as a class A indication. Individuals with higher surgical risks (e.g., $3 \%$ to $5 \%$ ), or less stenosis ( $40 \%$ to $60 \%$ ) even with suspected ulcerations, are contraindicated for intervention because the patients at high risk who would benefit from prophylactic intervention cannot be identified with fidelity using current imaging techniques. Indeed, stroke cost in the US continues around \$54 billion/year and is climbing.

Nanodiagnostics are highly suited to intravascular thrombus imaging, particularly for MRI and an emergent modality SPECTRAL CT. Spectral CT simultaneously acquires and presents both a traditional CT image and a K-edge contrast image superimposed, which uniquely differentiates the contrast material (e.g., ytterbium, gold, bismuth) from other 
attenuating materials, such as intraplaque calcium. We have developed and characterized fibrin-specific "soft nanoparticles" comprised of high-density organometallic complexes, which provide high SPECTRAL CT contrast against in vitro phantoms and iliac thrombus after intravenous injection and circulation in vivo (i.e., rabbits). These nanoagents were degraded in vivo and the heavy metal complexes bioeliminated from the body, as opposed to solid hard metal particles that can remain in the body indefinitely. Spectral CT could be used as a limited scan to rapidly evaluate carotid and vertebral atherosclerotic disease in patient suspected of or at high risk for embolic stroke.

021. NANOFORMULATED CELL PENETRATING SURVIVIN MUTANT AND LACTOFERRIN INDUCED DIFFERENTIATION AND P-GLYCOPROTEIN M E D I AT E D A N T I - E P IL EPT IC DRUG RESISTANCE REVERSAL

Kanwar JR ${ }^{1}$, Sriramoju B ${ }^{1}$, Neerati $\mathrm{P}^{2}$, Takkalapally $\mathrm{P}^{2}$, Kanwar RK ${ }^{1}$. ${ }^{1}$ Nanomedicine Laboratory of Immunology and Molecular Biomedical Research (NLIMBR), School of Medicine, Faculty of Health, Deakin University, Geelong Technology Precinct, Pigdons Road, Waurn Ponds, Geelong, Victoria 3217, Australia; ${ }^{2}$ Department of Pharmacology, University College of Pharmaceutical Sciences, Kakatiya University, Andhra Pradesh, Warangal 506002, India.

In this study, we investigated the differential actions of dominant negative survivin mutant (SurR9-C84A) against the cancerous SK-N-SH neuroblastoma cell lines and the differentiated SK-N-SH neurons. In both the cases the mutant protein displayed dual actions where its effects were cytotoxic towards cancerous cells and proliferative towards the differentiated neurons. In this study, we also investigated the neuronal differentiating actions of native bovine lactoferrin (bLf) and its iron saturated form (Fe-bLf). Both forms induced differentiation in the cancerous SK-N-SH neuroblastoma cells when treated at a concentration lower than the threshold dose required for anti-cancer activity. This was evident with the increased expression of neuronal specific differentiating markers (neurofilament (NF) 68, anti NF 160, anti-NF 200 etc). bLf adopted PI3K signalling predominantly for inducing differentiation while Fe-bLf showed these effects involving both PI3K and ERK pathways. However they showed a common ability in lowering the expression of endogenous survivin which is highly specific to the actively proliferating tumor cells. This is explained as lowered endogenous survivin expression in the SK-N-SH cells has halted their proliferative ability and induced the preliminary preparative changes required for differentiation. This phenomenon is in compliance with the fact that differentiating/differentiated cells lose their ability to divide and proliferate thus confirming the Lf differentiating effects. In addition, Fe-bLf showed significant in vitro P-gp inhibitory activity and hence was tested in an in vivo epileptic model. Epilepsy, one of the common chronic neurological disorders is characterized by frequent seizures and convulsions along with severe drug resistance due to the P-gp involvement. Surprisingly, in our study Fe-bLf significantly enhanced the phenytoin accumulation in the brain thereby its efficacy when given in combination. Thus, bLf entitles a novel therapeutic protein for treating brain tumors and neurodegenerative diseases with its differentiating effects while the P-gp inhibitory effects effects of Fe-bLf are highly useful in reversing the drug resistance as seen in epilepsy. In this study, we have also developed a novel PLGA nanoparticulate formulation to surmount the hurdles associated with the delivery of SurR9-C84A thus enhancing its effective therapeutic outcome.

\section{CHARACTERIZATION OF NANOPARTICLE COMPLEMENT INTERACTION}

Pham $\mathrm{CTN}^{1}$, Pan $\mathrm{D}^{2}$, Gordon $\mathrm{M}^{1}$, Lanza GM ${ }^{1}$, Hourcade $\mathrm{DE}^{1}$. ${ }^{1}$ Washington University in St. Louis and ${ }^{2}$ University of Illinois at Urbana-Champaign.

Nanoparticles offer new options for medical diagnosis and therapeutics with their capacity to specifically target cells and tissues with imaging agents and/or drug payloads. The unique physical aspects of nanoparticles present new challenges for this promising technology and raise additional safety issues arising from their interactions with blood borne components. Unlike small molecules, nanoparticles are subject to the scrutiny of host immune surveillance, the complement system in particular. Studies indicate that nanoparticles often elicit moderate to severe complement activation that may limit their clinical application. The complement system comprises three distinct pathways: the classical pathway (CP) is initiated by antibody:antigen complexes and the lectin pathway (LP) by polysaccharides; the alternative pathway (AP) functions spontaneously at low level and can serve to amplify the CP. Using parallel in vivo mouse models and an in vitro hemolysis-based assay we sought to define the pathways and key components involved in modulating complement interactions with perfluorocarbon nanoparticles (PFOBs). Our results indicate that in the mouse antibody-dependent recognition of certain PFOBs triggers $\mathrm{CP}$ activation, which is then amplified by the AP. To correlate results obtained in the mouse with the human system we employed a modified in vitro hemolysis-based assay to analyze nanoparticle-mediated complement activity in human sera against a panel of PFOBs and to examine variations between individuals. We found that nanoparticle surface charge/ chemistry/load all contribute to complement activation. We also found significant variations in nanoparticle:complement interactions in sera derived from individuals of different race, gender, and age. This dual approach, using in vivo mouse models and 
in vitro hemolysis-based assay, may provide the tools for an indepth structure-activity relationship study that will guide the development of biocompatible nanoparticles.

\section{ENDOTHELIAL NANOMEDICINE}

Muzykantov V. Department of Pharmacology, PENN School of Medicine, Smilow Translational Research Center, Philadelphia, PA 10104-5158.

Endothelium lining vascular lumen controls blood flow and pressure, as well as vascular permeability and transfer of circulating materials to tissues. Endothelial cells and their abnormalities play key role in cardiovascular, neurological, oncological and other disease conditions. They represent a key tripartite target, victim and barrier of nanomedicine. Drugs and carriers have no natural affinity to endothelium. Nanocarriers targeted to molecules expressed on the endothelial luminal surface optimize delivery of cargoes to and across these cells. We have devised a series of nanocarriers with diverse geometries (size, shape and plasticity) and affinity to specific endothelial epitopes. These features control key parameters of endothelial drug delivery: pharmacokinetics, binding to selected endothelial cell phenotypes, intracellular addressing and duration of therapeutic effects. Pathological factors (abnormal flow, inflammation) also regulate endothelial targeting and uptake of nanocarriers. Using these nanocarriers for endothelial delivery of antioxidant and anti-inflammatory agents provides therapeutic effects unattainable by nontargeted counterparts, motivating translational efforts in endothelial nanomedicine.

\section{4. ${ }^{64}$ CU-DOPED NANOPARTICLES FOR IN VIVO TUMOR IMAGING AND THERAPY}

Sun X, Huang X, Chen X. Laboratory of Molecular Imaging and Nanomedicine, National Institute of Biomedical Imaging and Bioengineering, National Institutes of Health, Bethesda, MD 20814.

Construction of self-illuminating semiconducting nanocrystals, also called quantum dots (QDs), has attracted much attention recently due to their potential as highly sensitive optical probes for biological imaging applications. Here we prepared a self-illuminating QD system by doping positron-emitting radionuclide $64 \mathrm{Cu}$ into $\mathrm{CdSe} / \mathrm{ZnS}$ core/shell QDs via a cation exchange reaction. The $64 \mathrm{Cu}-$ doped $\mathrm{CdSe} / \mathrm{ZnS}$ QDs exhibit efficient Cerenkov resonance energy transfer (CRET). The signal of $64 \mathrm{Cu}$ can accurately reflect the biodistribution of the QDs during the circulation with no dissociation of $64 \mathrm{Cu}$ from the nanoparticles. We also explored this system for in vivo tumor imaging. This nanoprobe showed high tumor targeting ability in a U87MG glioblastoma xenograft model and feasibility for in vivo luminescence imaging of tumor in the absence of excitation light. The availability of these self-illuminating integrated QDs provides an accurate and convenient tool for in vivo tumor imaging and detection.

Similar approach was also applied to label gold nanoparticles by chemically reducing $64 \mathrm{Cu}$ on the surface of $\mathrm{Au}$. This chelator-free method can be applicable to different $\mathrm{Au}$ nanomaterials regardless of the size, shape and surface coating. $64 \mathrm{Cu}$ integration has excellent stability with no dissociation of $64 \mathrm{Cu}$ from the particles during circulation and thus no loss of free $64 \mathrm{Cu}$ radioactivity into circulation and thus renal clearance. Compared with traditional $64 \mathrm{Cu}$ chelated nanomaterials, our $64 \mathrm{Cu}$ integrated nanomaterials can give more accurate evaluation of the pharmacokinetics after systemic administration. Using $64 \mathrm{Cu}$ labeled RGD peptide conjugated $\mathrm{Au}$ nanorods (NRs) with absorption wavelength around $808 \mathrm{~nm}$ (Au NR808) as an example, we further demonstrated the feasibility of these agents to target, monitor and treat tumors. This novel $64 \mathrm{Cu}$ labeling strategy will build a bridge between positron emission tomography (PET) imaging and therapeutic nanoagents.

\section{SESSION 5: NEUROREGENERATIVE MEDICINE: PROMISE AND PERILS}

\section{NANOTECHNOLOGY APPROACHES FOR CONTROLLING STEM CELL FATE}

Lee KB. Department of Chemistry \& Chemical Biology, Rutgers University, Piscataway, NJ 08854.

This presentation will focus on the interface between nanomedicine and stem cell biology. Even though it is wellestablished that stem cell fate is regulated by interactions that occur between microenvironmental cues and intrinsic cellular programs, our understanding of the function of the microenvironment and gene expression in stem cells is hampered by the limitations of conventional methods and the lack of extensive knowledge of multiple regulatory signals. If complex stem cell behaviors, such as differentiation and proliferation, are to be fully investigated, both approaches from nanotechnology - the "top-down" patterning of extracellular matrix (ECM) and signal molecules (e.g. ECM compositions, nanotopography, pattern geometry, and pattern density), and the "bottom-up" synthesis of multifunctional nanoparticles and their surface modification with specific signal molecules-should be combined synergistically. To address the aforementioned challenges, our research mainly focuses on two approaches: i) the development of combinatorial arrays of microenvironmental signal molecules for the investigation of stem cell behaviors; and ii) the synthesis and utilization of multifunctional nanoparticles as drug (e.g. small molecules or siRNA) and gene delivery vehicles to manipulate the expression of key genes in stem cells. 
More specifically, we have applied combinatorial signal arrays to study the temporal/spatial effect of microenvironmental cues on adhesion, growth, and differentiation of neural stem cells. Furthermore, novel multifunctional magnetic nanoparticles were synthesized and utilized to deliver genetic materials (e.g. siRNA and plasma DNA) into stem cells and for multimodal imaging for controlling their neurodifferentiation pathways. In this presentation, a summary of the most updated results from these efforts and future directions will be discussed.

\section{CELL-TOPOGRAPHY INTERACTIONS AND NEURONAL DIFFERENTIATION}

Kulangara K, Adler AF, Grigsby CL, Yim EKF, Leong KW. Department of Biomedical Engineering, and Surgery, Duke University, Durham, NC 27705.

Topography of extracellular microenvironment can influence cellular responses from attachment and migration to differentiation and production of new tissue. Cells in their natural environment interact with extracellular matrix that contains structures in the nanometer scale. Nanoscaled topography of synthetic materials, through its resemblance to in vivo surroundings, may provide potent cues to influence the phenotypes of cells seeded on these nanostructured biomaterials. This presentation will highlight our recent work on understanding the mechanism of cell-topography interactions and exploiting these interactions for modulating direct cellular reprogramming.

\section{NEUROPROTECTIVE REGULATORY T CELLS AS VEHICLES FOR NANOFORMULATED GROWTH FACTOR DELIVERY FOLLOWING TRAUMATIC BRAIN INJURY}

Kelso $\mathrm{M}^{1}$, Elliott $\mathrm{BE}^{2}$, Gendelman $\mathrm{HE}^{2}{ }^{1}$ College of Pharmacy, Pharmacy Practice and ${ }^{2}$ Department of Pharmacology and Experimental Neuroscience, University of Nebraska Medical Center, Omaha, NE 68198.

Traumatic brain injury (TBI) affects neural cells and cerebral vasculature integrity leading to progressive neurodegeneration. The innate and adaptive immune systems respond to such injury leading to facilitated damage and repair. The balance between $\mathrm{T}$ effector (Teff) and regulatory $\mathrm{T}$ cell (Treg) functions can regulate neuroinflammatory responses at sites of tissue damage. Here, Teff accelerate while Treg restrict continued tissue damage. These actions suggest that Tregs may be harnessed to positively affect disease outcomes and for targeted drug delivery. To facilitate a Treg protective immune response, we subjected C57BL/6 mice to a controlled cortical impact. The mice were randomized to receive either granulocyte macrophage colony stimulating factor (GM-CSF; $50 \mu \mathrm{g} / \mathrm{kg}$ ), a hematopoietic stem cell factor and known Treg inducer, or an equal volume of saline $6 \mathrm{~h}$ after injury then continued daily until sacrifice ( 7 or 14 days). GM-CSF modestly increased splenic Treg populations. Sparing of cortical tissue was seen after GM-CSF administration with untreated animals having lesions $\sim 10-15 \%$ larger than treated animals. Staining for ionized calcium binding adaptor molecule 1 , a microglia marker, was seen at the lesion site as well as the surrounding regions on days 7 and 14. GM-CSF significantly decreased staining and appeared to alter microglia morphology. Immunoreactivity for glial fibrillary acidic protein, an astrocyte marker, did not differ amongst treatment groups. As GM-CSF administration can elicit neuroprotection, we theorized the Treg could be used as a drug carrier and notably for vascular endothelial growth factor (VEGF) which shows angiogenic and neuroprotective properties following TBI. Therefore, we hypothesized that $\mathrm{T}$ cell targeted delivery of a VEGF complex prepared using anionic block copolymers of poly(ethylene oxide)-block-poly(L-glutamic acid) could provide synergistic protection to an injured vasculature and parenchyma.

Supported by: Carol Swarts Neuroscience Research Laboratory, the Frances and Louie Blumkin Foundation, Department of Defense Grant W81XWH11-1-0700, and National Institutes of Health grants P20 GM103480, P01 DA028555, R01 NS36126, P01 NS31492, 2R01 NS034239, P01 MH64570 and P01 NS43985.

\section{PERSPECTIVE FOR NANOTOXICOLOGY}

Toborek M. Department of Biochemistry and Molecular Biology, University of Miami School of Medicine, Miami, FL 33136.

Various nanotechnology applications and engineered nanomaterials (ENMs) have been introduced for treatment, diagnosis, monitoring, and control of biological systems. However, surprisingly, remarkably little is known about the health hazards of ENMs. This lack of knowledge creates a significant impediment for accurately assessing the risks of ENM exposures and setting exposure standards that are safe for public health. Potential toxic effects of ENMs are an emerging concern in medicine and toxicology. The complexity of the ENM surface offers physico-chemical properties that are substantially different from the bulk material and may contribute to their toxicity and health risk. These properties are further altered by surface modifications of ENMs, which occur due to contact with biological fluids or tissues. We have evidence that binding of organic pollutants (namely, polychlorinated biphenyls; PCBs) to seemingly inert silica nanoparticles markedly potentiate vascular toxicity of PCBs. In addition, we evaluated toxicity of nanoalumina, which is among the most abundant chemicals produced as nanosized particles, estimated to account for $\sim 20 \%$ of the world market of ENMs. Treatment of brain endothelial cells with nanoalumina induced in dose- 
dependent mitochondrial potential collapse, increased autophagy, and decreased expression of tight junction proteins, occludin and claudin-5. Systemic treatment with nanoalumina resulted in early localization to the brain endothelium, followed by an entry into the brain parenchyma. Nanoalumina readily accumulated in the brain and was not eliminated from the brain components, causing disruption of the blood-brain barrier permeability and facilitating stroke development. Overall, these results caution about the nanoneurotoxicity of ENMs, indicating that they accumulate in the brain and may induce previously unforeseen neurotoxic effects.

\section{GENE THERAPY APPROACHES TO REGENERATING THE OPTIC NERVE}

Foldvari M and Alqawlaq S. School of Pharmacy, Waterloo Institute for Nanotechnology, University of Waterloo, Waterloo, Canada.

Glaucoma is the second leading cause of blindness with 60.5 million patients worldwide in 2010, a figure that is expected to increase to 79.6 million by 2020 . Gene therapy with neurotrophic factors has potential to provide neuropreventative and neuroregenerative functions for retinal ganglion cells (RGCs). However, a significant limitation in ophthalmology is the lack of effective delivery systems to the retina and the optic nerve. 'Effective' encompasses several qualitative, quantitative and safety requirements. These are: 1 ) efficiency of delivery to the back of the eye; 2) biocompatibility; 3 ) targeted function; 4) prolonged effect; and 5) potentially non-invasive, pain-free and low-risk administration. Currently, intravitreal and subretinal injections are used for reaching the RGCs. Ultimately, topical administration to the ocular surface would be the safest method for gene delivery to the retina, since it is non-invasive and painless compared to other delivery methods. We have developed and characterized non-viral gemini surfactant-phospholipid nanoparticles (GL-NPs) for intravitreal and topical administration. GL-NPs carrying Cy5-labeleld plasmid DNA demonstrated distinct trafficking behaviour and biodisposition within the eye in vivo in mice after intravitreal or topical application with respect to pathways of movement and physicochemical stability. An overview will be provided on the design and formulation of gemini NPs as gene carriers, NP toxicity and the identification of parameters that require deeper understanding to develop successful topical gene delivery systems targeting the retina.

\section{SESSION 6: BENCH TO BEDSIDE: APPLICATIONS FOR NANOMEDICINES}

\section{CARRIERS THAT BREAK BARRIERS}

Batrakova $\mathrm{E}^{1}$, Haney $\mathrm{MJ}^{1}$, Zhao $\mathrm{Y}^{1}$, Harrison $\mathrm{EB}^{2}$, Mahajan $\mathrm{V}^{1,3}$, Ahmed $\mathrm{S}^{2}$, He $\mathrm{Z}^{1}$, Suresh $\mathrm{P}^{2,3}$, Hingtgen $\mathrm{SD}^{1}$, Klyachko $\mathrm{NL}^{1,5}$, Mosley $\mathrm{RL}^{3}$, Gendelman $\mathrm{HE}^{3}$, Kabanov $\mathrm{AV}^{1,4}$.
${ }^{1}$ Eshelman School of Pharmacy, University of North Carolina at Chapel Hill, Chapel Hill, NC 27599-7362; ${ }^{2}$ Department of Pharmaceutical Sciences, Center for Drug Delivery and Nanomedicine, ${ }^{3}$ Department of Pharmacology and Experimental Neuroscience, University of Nebraska Medical Center, Omaha, NE 68198; and ${ }^{4}$ Deparment of Chemical Enzymology, Faculty of Chemistry, M.V. Lomonosov Moscow State University, Moscow, Russia.

The ability to precisely upregulate genes in inflamed brain holds great therapeutic promise. Here we report a novel class of vectors, genetically modified macrophages that carry reporter and therapeutic genes to neural cells. Systemic administration of macrophages transfected ex vivo with a plasmid DNA (pDNA) encoding a potent antioxidant enzyme, catalase, produced month-long expression levels of catalase in the brain resulting in three-fold reductions in inflammation and complete neuroprotection in mouse models of Parkinson's disease (PD). This resulted in significant improvements in motor functions in PD mice. Mechanistic studies revealed that transfected macrophages secreted extracellular vesicles, exosomes, packed with catalase genetic material, pDNA and mRNA, active catalase, and NF- $k b$ a transcription factor involved in the encoded gene expression. Exosomes efficiently transfer their contents to contiguous neurons resulting in de novo protein synthesis in target cells. Thus, genetically modified macrophages serve as a highly efficient system for reproduction, packaging, and targeted gene and drug delivery to treat inflammatory and neurodegenerative disorders.

\section{MITOCHONDRIA AT THE ORIGIN OF OXIDA- TIVE STRESS}

Perry $\mathrm{G}^{1,2}$, Wang $\mathrm{X}^{2}$, Moreira $\mathrm{P}^{3}$, Castellani $\mathrm{RJ}^{4}$, Nunomura $\overline{\mathrm{A}^{5}, \mathrm{Zhu} \mathrm{X}^{2}} .{ }^{1}$ College of Sciences, University of Texas at San Antonio, San Antonio, TX; ${ }^{2}$ Case Western Reserve University, Cleveland, Ohio, ${ }^{3}$ University of Coimbra, Portugal, ${ }^{4}$ University of Maryland School of Medicine, ${ }^{5}$ University of Yamanashi, Japan.

Mitochondria may underlie oxidative stress in Alzheimer disease (AD) changes since dysfunction is a prominent and early feature of $\mathrm{AD}$. Recent studies demonstrate that mitochondria are dynamic organelles that undergo continual fission and fusion events, which regulate their morphology and distribution. Morphometry showed a small but significant reduction in mitochondria number and enlarged size in $\mathrm{AD}$. Levels of the fission/fusion proteins DLP1, OPA1, Mfn1 and Mfn2C were significantly decreased in $\mathrm{AD}$, yet levels of Fis 1 were significantly increased. Interestingly, although all these proteins demonstrate even distribution in the cytoplasm and processes of pyramidal neurons in age-matched control hippocampus, they appeared to accumulate in the soma but not in the processes of pyramidal neurons in AD hippocampus. Given that 
OPA1, Fis1, and Mfn1/2 are all mitochondrial membrane proteins, the changes in their distribution to soma in $\mathrm{AD}$ neurons, suggest changes in mitochondria distribution in these neurons. The expression of fission/fusion proteins was manipulated in M17 cells and primary hippocampal neurons in a way that mimicked their expression changes in AD. These manipulations all reduced mitochondrial density in the cell periphery (M17 cells) or neuronal processes (primary neurons) which correlated with reduced spine numbers (primary neurons).

AbetaPP and Abeta caused reduced expression of DLP1 and OPA1 while increasing expression of Fis1, consistent with our findings in AD brains. Through time lapse study, we were able to demonstrate that mitochondria were able to fuse with each other but at a much slower rate in AbetaPP overexpressing cells.

Overall, we concluded that AbetaPP, through Abeta production impairs mitochondrial fission/fusion balance through regulation of expression of mitochondria fission and fusion proteins.

Supported by: NIH, National Institute on Minority Health and Health Disparities G12MD007591.

\section{NANOMEDICINES FOR NEUROPROTECTION AND NEUROREGENERATION}

Mallapragada SK, Sharma A, Zbarska S, Brenza T, Ghasias S, Anantharam V, Narasimhan B, Sakaguchi D, Kanthasamy A. Department of Chemical and Biological Engineering, Iowa State University, Ames, IA 50011-2230.

In many neurodegenerative diseases such as Parkinson's (PD) and Alzheimer's (AD), or with traumatic brain injuries, there is a progressive loss of structure or function of neurons, including death of neurons. Mitochondrial dysfunction, oxidative and nitrative stresses have been implicated in number of neurodegenerative conditions including PD and AD. Mitochondrial targeted antioxidants have been recently shown to protect against Parkinsonian toxicant, methyl-4phenylpyridinium $(\mathrm{MPP}+)$-induced production of reactive oxygen and nitrogen species production and apoptotic cell death. Herein, we have investigated the ability of biodegradable polyanhydride nanoparticles to enhance the delivery of mitochondrial targeted antioxidants to protect against MPP+ induced dopaminergic neurotoxicity. The antioxidant utilized in these studies apocynin was functionalized with a mitochondrial targeting ligand to yield mitoapocynin. Using polyanhydride nanoparticles, we designed a delivery system for targeted release of mitoapocynin to mitochondria of cells. The performance of the designed delivery system was evaluated for neuroprotection against MPP+-induced neurotoxic cell death in both dopaminergic cell lines and mouse primary neurons. Cellular uptake and intracellular trafficking of the particles was evaluated. In a parallel approach, nanomedicine- based strategies can significantly impact peripheral nerve regeneration as well. Schwann cells, in conjunction with micropatterned substrates, have been shown to secrete nerve growth factor and facilitate peripheral nerve regeneration. In order to facilitate peripheral nerve regeneration, polymeric conduits with micro/nanopatterns were fabricated and the transdifferentiation of mesenchymal stem cells to Schwann-cell like phenotypes was investigated. This talk will therefore highlight the importance of nanomedicine in both peripheral and central nervous system regeneration.

Supported by: US Army Grant No. W81XWH-10-1-0806

\section{ULTRASMALL HYBRID INORGANIC NANOPAR- TICLE PROBES FOR BRAIN TUMOR IMAGING}

Bradbury $\mathrm{M}^{1}$, Zanzonico $\mathrm{P}^{2}$, Larson $\mathrm{S}^{1}$, Wiesner $\mathrm{U}^{3}$. ${ }^{1}$ Department of Radiology and ${ }^{2}$ Department of Medical Physics, Sloan Kettering Institute for Cancer Research, New York, NY 10065; ${ }^{3}$ Department of Materials Science and Engineering, Cornell University, Ithaca, NY 14850.

Despite recent advances in imaging probe development for nanomedicine, the translation of targeted diagnostic platforms remains challenging. Nanomaterials platforms currently under evaluation in oncology clinical trials are largely non-targeted drug delivery vehicles that not typically surface modified for direct detection by clinical imaging tools. Here we developed renally-excreted, fluorescent (Cy5 dye) hybrid silica particle probes (Cornell or $\mathrm{C}$ dots) that have been tailored to specific molecular targets (i.e., integrins) and labeled with radioactive iodine moieties. This PET-optical particle imaging probe, which has received FDA investigational new drug application (IND) approval, may lead to improvements in cancer detection, staging, and treatment management in humans. Coupled with PET imaging and analysis tools, complete and quantitatively accurate data sets for targeting kinetics, whole body distributions, and clearance profiles have been acquired in preclinical tumor models, as well as in early phase first-inhuman trials following intravenous tracer administration. Findings in humans are consistent with a well-tolerated inorganic particle tracer, which exhibits in vivo stability and distinctly unique and reproducible pharmacokinetic signatures defined by renal excretion. The preferential uptake and localization of the probe at sites of integrin-expressing lesions, including those within the central nervous system, suggest the potential utility of these targeted $\mathrm{C}$ dots in cancer diagnostics. We further show the use of these methods to accurately estimate the fraction of the injected particle load that accumulates at sites of brain tumors, in addition to monitoring timedependent changes in particle uptake. The foregoing information can be extended to treatment management settings for estimating particle dosing requirements in the context of therapeutic interventions. 


\section{TARGETING NEURONS WITH CHITOISAN NANOPARTICLES}

Gabriel Lopez-Berestein, MD, University of Texas MD Anderson Cancer Center, Houston, TX 77030.

Dr. Lopez-Berestein's research efforts concentrate on the development of nanoliposomes and nanoparticles for the delivery of siRNA to target major signaling pathways. Several of these targets were identified to play a prominent role in cell growth in his laboratory. He brought from concept to clinic, seven different antimicrobial and antitumor agents. Of those seven, one, Abelcet, reached the market and the other six continue in different stages of clinical development. For this presentation, he will speak on targeting neurons with chitoisan nanoparticles.

\section{NANOFIBER AND NANOCARRIER MEDIATED MODULATION OF CELL MIGRATION FOR NERVE REPAIR AND BRAIN TUMOR THERAPY}

Bellamkonda R. Department of Biomedical Engineering, Georgia Institute of Technology and Emory School of Medicine, Atlanta, CA 30332.

Control of cell migration is important for healing of damaged tissues and on the flip side, cell invasion is at the root of the challenge that clinicians face in treating brain tumors. In my laboratory we have been exploring the use of thin film mediated topographical cues to enable efficient and controlled migration of cells. In the peripheral nerve context, induction of Schwann cell migration into critically sized nerve gaps enables bridging of long nerve gaps.

In the context of brain tumors, we are designing novel nanocarriers that inhibit brain tumor invasion and demonstrate that controlling tumor invasion renders gliomas vulnerable to approved chemotherapeutics.

We are also designing devices to control the invasion of brain tumors along paths that we specify using topographical guidance of brain tumors in vivo. We demonstrate, for the first time to our knowledge, that topographical cues presented by thin films enable moving a primary tumor from an intracortical region to an extracortical hydrogel sink where the tumor cells are killed. This novel approach of bring the tumor to the drug rather than the drug to the tumor is enabled by our ability to design constructs that enable controlled, directional migration of invasive brain tumors.

\section{SESSION 7: EARLY CAREER INVESTIGATORS}

\section{NANOMEDICINE: AN EDITOR'S VIEW \\ Balogh L. Editor-in-Chief, Nanomedicine NBM (Elsevier).}

Nanomedicine and nanobiotechnology are two rapidly emerging interdisciplinary areas creating novel materials, substances, and procedures useful for medicine and life sciences in general. Nanomedicine focuses on the diagnosis, treatment, monitoring of patients as well as on the prediction and prevention of disease and has the potential to forever change how patients are diagnosed and treated in the next decade. However, all of this is happening when both medical science and the pharmaceutical industry are undergoing radical transformations. There are many challenges for these paradigm-changing fields, especially in the area of scientific communication and commercialization. This talk will focus on scientific publishing. This field is also undergoing dynamic changes, the essential general question being how to determine value of research before and/or after making it public, and how to monetize this value. This discussion has now expanded to the whole society and several articles have recently appeared in well-known journals (Science, Nature, The Economist, etc.) questioning the value and methods of science.

Getting published is crucial for academicians and researchers. In this talk the speaker will summarize major changes in business models (traditional, open access, and hybrid), describe the use of Impact Factor, and introduce the latest scientific methods used to determine the value of journals, and explain how these tools may be used correctly to evaluate individual authors, groups, institutions, and countries. In addition to comparing leading nanomedicine journals, a Q\&A opportunity will also be provided.

\section{NANOMEDICINE AND TOXICOLOGY}

Peterson E. Department of Pharmacology/Toxicology, University of Arkansas for Medical Sciences, Little Rock AR 72205 .

Methamphetamine (METH) and related stimulant abuse is one of the most significant drug problems in the US and abroad. There are no FDA approved medications to treat METH addiction. Biologic-based medications such as anti-METH monoclonal antibodies and their sequence derivatives are showing promise in preclinical studies. Both a long-acting $\operatorname{IgG}$ and extremely short-acting single chain variable fragment $(\mathrm{scFv})$ have demonstrated preclinical efficacy. While the scFv has important advantages over IgG in terms of cost, ease of production, and dose, the in vivo half-life is too short to offer longterm protection from METH. Thus, to improve pharmacokinetics and increase binding properties, we conjugated anti-METH scFvs to dendrimer nanoparticles to generate nanoconjugates termed dendribodies. The anti-METH dendribodies retain high affinity METH-binding properties in vitro and in vivo. Moreover, in rat studies we found that conjugating the anti-METH scFvs to dendrimers resulted in an extension of the serum halflife from $1.3 \mathrm{~h}$ for the $\mathrm{scFv}$ to $26 \mathrm{~h}$ for the dendribodies. This represents a 20-fold increase in the duration of action. Thus, the dendribody design is a potential platform for generating multivalent products from antibody fragments that could translate to medications with customizable pharmacokinetic profiles.

Supported by: NIH, NIDA R01 DA026423 
038. NANOPARTICLE INDUCED ENDOTHELIAL LEAKINESS MECHANISM AND IMPLICATIONS TO NANOMEDICINE

Setyawati MI and Leong DT. Department of Chemical and Biomolecular Engineering, National University of Singapore 117585.

The endothelium is a common tissue that most nanomedicine would interact with. While it is not usually the target tissue of action, how nanomaterials interact with the endothelium is an important factor to consider. We have shown that inorganic nanomaterials (Ag, Silica, Titanium Dioxide of primary size 15-30 nm) caused endothelial leakiness (both in vitro and in vivo). The mechanism was that these nanomaterials enter and bind to VE-cadherin found in the adherens junctions between endothelial cells and disrupted the junction, leading to intracellular signaling that led to a leakiness phenotype and we coined this as Nanoparticle induced endothelial leakiness (NanoEL) (Setyawati et al. Nature Comms 2013, 4, 1673). We also showed that cell tension plays a major part in this disruption and relaxing cell tension with inhibitors greatly reduce the NanoEL effect in endothelial cells (unpublished data). We have also worked out nanoparticle centric parameters that determine NanoEL so that the nanomedicine field can have design rules to either induce or avoid NanoEL (unpublished data). The overall impact of NanoEL to nanotechnology is that it might be the real nanoparticle induced effect hidden under the guise of the EPR effect (invited perspective manuscript in prep).

039. "METABOLIC PHENOTYPING TO FUNCTIONALLY CHARACTERIZE BRAIN REGIONS"

$\underline{\text { Ivanisevic } \mathrm{J}^{1}}$, Epstein $\mathrm{AA}^{2}$, Kurczy $\mathrm{M}^{1}$, Benton $\mathrm{P}^{1}$, Boska $\mathrm{MD}^{3}$, Fox HS${ }^{2}$, Gendelman $\mathrm{HE}^{2}$, Siuzdak $\mathrm{G}^{1}$. ${ }^{1}$ The Scripps Research Institute, CA 92037; ${ }^{2}$ Department of Pharmacology and Experimental Neuroscience, ${ }^{3}$ Department of Radiology, University of Nebraska Medical Center, Omaha, NE 68198.

Historically, the studies of brain metabolism and the related pathological changes are based on targeted analysis of limited number of metabolites. Here we use mass spectrometry based untargeted metabolomics to examine the metabolic balance across different regions of brain in a humanized mouse model. Liquid Chromatography Mass Spectrometry (LC/MS) and Nanostructure Imaging Mass Spectrometry (NIMS) revealed significant variations in water soluble and in lipid brain metabolome between different regions. Differences in abundance were observed for some of the major brain neurotransmitters and characteristic phospholipid metabolites. This study shows that untargeted metabolic profiling can provide a new insight into functional differences between brain regions, including discovery of unanticipated roles of specific metabolites, and localization of metabolic changes that correlate to neurocognitive disorders.

\section{TRANSPORT OF ICAM-1-TARGETED NANOPARTICLES ACROSS ENDOTHELIAL- SUBENDOTHELIAL LAYERS AND UPTAKE BY NEURONS}

$\underline{\text { Hsu J }}^{1}$, Rappaport $\mathbf{J}^{1}$, Muro $\mathrm{S}^{1,2}$. ${ }^{1}$ Fischell Department of Bioengineering and ${ }^{2}$ Institute for Biosciences and Biotechnology Research, University of Maryland, College Park, MD 20742.

Systemic drug delivery into the brain is impeded by the blood-brain barrier (BBB), a lining of endothelial cells (EC) and subendothelial cells, astrocytes (AC) and pericytes (PC) that regulates transport between the bloodstream and neuronal cells (NC) in the brain. Targeting EC surface receptors involved in transcytosis offers an avenue to traverse this barrier. Intercellular adhesion molecule 1 (ICAM-1), a molecule involved in leukocyte extravasation, can transport ICAM-1targeted particles across cell monolayers, but their interactions with BBB and brain cells have not been tested. We used fluorescence microscopy and radioisotope tracing to explore transport of $100 \mathrm{~nm}$ polymer particles coated with antibodies to ICAM-1 (anti-ICAM NP) into and/or across EC, AC, PC, and $\mathrm{NC}$, cultured as monocultures or endothelial+ subendothelial co-cultures. Under pathological-like conditions, AC and PC expressed ICAM-1, but at lower levels than EC ( $78 \%$ and $54 \%$ of EC). As a result, AC and PC bound and internalized anti-ICAM NP mainly by the CAM pathway (not clathrin pits or caveoli), yet, at a lower level than EC: by $1 \mathrm{~h}$, $\mathrm{AC}$ and PC bound 79 and $95 \mathrm{NP} /$ cell vs $200 \mathrm{NP} /$ cell for EC, and internalized 42 and $43 \mathrm{NP} /$ cell vs $110 \mathrm{NP} /$ cell for EC. This suggests that BBB ICAM-1 is more accessible to targeting from the endothelial side, as required for systemic administration, and retention within subendothelial cells may be low, allowing penetration into the brain. Accordingly, anti-ICAM $\mathrm{NP}$ targeted brain EC 19-fold over IgG NP and $2.3 \times 10^{\wedge} 6 \mathrm{NP} /$ $\mathrm{mm}^{\wedge} 2$ were transported across EC monolayers by $5 \mathrm{~h}$. AntiICAM NP transported across the EC lining were taken up by subendothelial $\mathrm{PC}$ or $\mathrm{AC}$ in co-cultures, resulting in transport of $1.2-3.4 \times 10^{\wedge} 6 \mathrm{NP} / \mathrm{mm}^{\wedge} 2$ across both layers by $5 \mathrm{~h}$. Also, NC expressed ICAM-1 and supported binding of $130 \mathrm{NP} /$ cell and uptake of $40 \mathrm{NP} /$ cell by $5 \mathrm{~h}$. Together, these results suggest that ICAM-1-targeted NP have the potential to improve drug delivery across the BBB and into neurons.

\section{SELF-ASSEMBLED LIPID-POLYMER HYBRID NANOPARTICLES FOR THE SUSTAINED DELIVERY OF SMALL INTERFERING RNA}

Zhu X, Shi J, Farokhzad OC. Laboratory of Nanomedicine and Biomaterials, Department of Anesthesiology, Brigham and Women's Hospital, Harvard Medical School, Boston, MA 02115. 
RNA interference (RNAi) has shown great potential for the treatment of various diseases including cancer. Numerous nanoparticle (NP) platforms, such as lipoplex and polyplex, have been developed to facilitate the safe and effective delivery of small interfering RNA (siRNA) - a major hurdle for the clinical applications of RNAi. Nevertheless, these NP systems lack the sustained siRNA release property, and thus can only induce transient gene silencing due to the short lifetime of siRNA. Therefore, the delivery of siRNA using controlledrelease NPs would be necessary for achieving sustained gene silencing. Herein, we develop a novel self-assembled lipidpolymer hybrid NP platform for safe and effective siRNA delivery in a sustained manner. The hybrid NPs show excellent in vitro knockdown efficacy at low doses of siRNA, and promising in vivo results for delivering siRNA to xenograft tumors. More importantly, these NPs can control the release of siRNA, with the half-release time of $\sim 9$ days, for sustained silencing of target gene expression. Results demonstrate that the expression of target proteins (e.g., Survivin and PHB1) can be completely silenced in 2 weeks after the cancer cells were transfected with the NPs for $6 \mathrm{~h}$. As a comparison, the protein expression is gradually recovered at day $7-14$, after cell transfection with lipo2000-siRNA complexes. Furthermore, this NP platform can be applied to co-deliver chemotherapeutics and siRNAs for the synergistic cancer treatment. We believe that the lipid-polymer hybrid NP platform with the property of sustained siRNA release could hold potential in both fundamental studies and clinical applications.

\section{SESSION 8: NANOMEDICINE FOR HIV ERADICATION IN BRAIN AND OTHER RESERVOIRS}

\section{SUSTAINED RELEASE FORMULATIONS FOR HIV THERAPY}

Owen A and Rannard S. University of Liverpool, Liverpool L693GF, UK.

Antiretroviral Therapy involves co-administration of multiple drugs to simultaneously inhibit viral targets, maximizing inhibition of replication while minimizing resistance. Antiretrovirals also have application in prevention of HIV infection and pre-exposure prophylaxis (PrEP) strategies have been investigated for subjects with high risk of acquiring infection. Several issues can jeopardize efficacy of HIV drugs and multiple factors contribute to heterogeneity in response to therapy. Currently available oral formulations necessitate lifelong, daily dosing and suboptimal adherence places patients at risk of treatment failure and low rates of protection for PrEP. Broadly, there are two nanotechnology approaches to efficacy improvement and enhanced formulation of hydrophobic or poorly soluble drugs. These are a) "nanocarriers" - utilizing materials science to attach or encapsulate drugs in a transport vehicle; and b) "nanoparticle-engineering" - forming a particle dispersion where each sub-micron particle consists of drug. Nanomilling has been the most successful nanoparticleengineering technology commercially, and relies on the formation of solid drug nanoparticles - ie particles made from drug with no carrier. Recently, nanomilling has generated sustained release antiretroviral formulations via intramuscular depot administration. Long-acting parenteral formulations of S/GSK744 and rilpivirine have been reported, for which plasma concentrations remain above the IC90 for 1-3 months after dosing. The advent of long-acting formulations has generated considerable excitement and a recent study of the interests and attitudes indicated that $84 \%$ of surveyed patients would definitely or probably try a once monthly nanoformulated antiretroviral therapy. These formulations represent extremely important progress for HIV therapy, and this presentation will provide an overview of current progress with a perspective on knowledge gaps and future development opportunities.

\section{A NANOPARTICLE APPROACH TO TARGETING LATENT HIV RESERVOIR}

Marsden $\mathrm{MD}^{1}$, Kovochich $\mathrm{M}^{1}$, Buehler DC ${ }^{1}$, Shen $\mathrm{S}^{1}$, Toso $\mathrm{DB}^{1}$, Wu $\mathrm{X}^{1}$, Loo JA ${ }^{1}$, Zhou $\mathrm{ZH}^{1}$, Kickhoefer $\mathrm{VA}^{1}$, Wender $\mathrm{PA}^{2}$, Rome LH ${ }^{1}$, Zack JA ${ }^{1} .{ }^{1}$ David Geffen School of Medicine, University of California, Los Angeles, Los Angeles, CA 90095; ${ }^{2}$ Stanford University, Stanford CA 94305.

Antiretroviral therapy can reduce HIV viremia to undetectable levels, but it does not eliminate infection. Rather a small, but very stable pool of latently infected memory CD4+ T cells remains, which upon cessation of therapy, rekindles productive viral infection. Thus to truly cure HIV disease, this reservoir must be eliminated. Unfortunately, latently infected T cells do not generally produce viral proteins and are thus not killed by the virus infection itself, and are indistinguishable from uninfected cells by the immune system. One potential means of eliminating this reservoir is to activate the latent virus while the patient is on therapy, which would induce viral gene expression and render the cell susceptible to virusmediated or immune-mediated killing. We have previously shown that protein kinase $\mathrm{C}$ (PKC) activators can induce viral gene expression from latently infected cells, and render these cells susceptible to targeting with an HIV Env-specific immunotoxin. However systemic delivery of these activators would likely result in unwanted side effects. Consequently we have chosen to pursue a targeted nanoparticle approach, to deliver activating molecules more specifically to $\mathrm{T}$ cell populations that might be infected. Our previous work established that the PKC activator bryostatin 1 can be packaged into liposomes, which can be targeted specifically to CD4 T cells by incorporating anti-CD4 antibodies onto the surface of the liposome. To potentially improve this type of strategy, we are bioengineering natural, self-assembling vault nanoparticles in 
a manner that allows them to reversibly encapsulate lipophilic compounds, such as bryostatin 1 . These particles are able to activate viral gene expression from latently infected cell lines, and we are currently assessing the ability of this approach to activate lymphocytes in vitro and in vivo.

\section{NANOTHERAPIES: CURRENT AND FUTURE CHALLENGES}

Schwartz $\mathrm{SA}^{1}$, Mahajan $\mathrm{S}^{1}$, Reynolds $\mathrm{JL}^{1}$, Ravikumar Aalinkeel $^{1}$, Nair $\mathrm{B}^{1}$, Dai L ${ }^{1}$, Mammen $\mathrm{M}^{1}$, Sykes $\mathrm{D}^{1}$, Prasad $\mathrm{P}^{2}$, Sellito $\mathrm{SJ}^{3}$, Cwiklinski $\mathrm{C}^{3}$. ${ }^{1}$ Department of Medicine, Division of Allergy, Immunology, \& Rheumatology and ${ }^{2}$ Institute for Lasers, Photonics and Biophotonics, Department of Chemistry, University at Buffalo, The State University of NY, Buffalo, NY 14260; ${ }^{3}$ Ceno Technologies, Inc. \& Small Matter, LLC, Buffalo, NY 14209.

Our lab has been using nanoparticles to optimize the delivery of several drugs and biological agents (e.g. siRNA) for the treatment of a variety of diseases. We explored nanotherapy for such diverse disorders as neuroAIDS, prostate cancer, drug addiction, asthma, food allergies, and neurodegenerative diseases. Several of our studies using nanoparticles to transport drugs across the blood-brain barrier (BBB) utilized unique models of the BBB including a hollow fiber culture system as a 3 dimensional example of the BBB. Our earlier investigations utilized quantum dots and gold nanoparticles which showed promise as theranostic agents. However concerns were raised about the long term retention of these nanoparticles in the body and their potential toxicities. Other nanoparticles that we evaluated for clinical application include chitosan, cationic liposomes, halloysites, and single walled carbon nanotubes. Current efforts are focused on biocompatible and biodegradable, nanoparticles composed of functionalized, cationic, polylactic acid. We also examined exosomes as cell or tissue specific, therapeutic delivery systems. This presentation will describe our studies and some of the hurdles that we encountered and their potential solutions in developing optimal nanotherapies.

\section{NANOFORMULATED ANTIRETROVIRAL THERAPYAND THE CNS}

Gendelman $\mathrm{HE}^{1}$, McMillan $\mathrm{J}^{1}$, Boska $\mathrm{M}^{2}$, Liu $\mathrm{Y}^{2}$, Poluektova $\mathrm{L}^{1}$, Gorantla $\mathrm{S}^{1}$, Bronich $\mathrm{T}^{3}$, Alnouti $\mathrm{Y}^{3}$, Liu $\mathrm{X}^{1}$. ${ }^{1}$ Department of Pharmacology and Experimental Neuroscience, ${ }^{2}$ Department of Radiology, ${ }^{3}$ College of Pharmacy, Pharmaceutical Science, University of Nebraska Medical Center, Omaha, NE 68198.

Drug toxicities, patient compliance and limited penetrance into viral reservoirs have diminished long-term antiretroviral therapy (ART) efficacy. Novel strategies serve to improve ART delivery to mononuclear phagocytes (MP: monocytes and tissue macrophages) and CD4+ T lymphocytes to bring drugs to viral reservoirs. Over the past decade we pioneered the development of long-acting injectable nanoformulated ART (nanoART) with superior pharmacokinetics. A "Trojan Horse" MP carriage of the drug formulations facilitated the development of long-lived storage depots, in liver and spleen. Our drug formulations enable drug transfer to an infected CNS. We are currently improving nanoART technology to move it to human use. We maximized viral clearance by developing a range of antiretroviral nanoformulated drugs. Synthesis of polymer drugs with attachments of specific ligand coating directed drugs to specific virus' target cells and subcellular regions to optimize antiretroviral activities. We probed directed formulations in rodents using a newly discovered small magnetite ART (SMART) platform. SMART formulations were synthesized by crystalline particles and evaluated for drug pharmacokinetics. Histologic and imaging assays evaluated drug toxicities. Drug polymer formulations were tested for antiretroviral efficacy, immune and neuroprotection in a humanized mouse model of HIV infection. The work improves nanoART towards the goal of viral eradication.

Supported by: NIH R01 AG043540-01A1, P01 DA02855504, R01 NS3439-19 (HEG).

\section{APPLICATION OF TARGETED NANO-NRTI COCKTAILS AGAINST HIV INFECTION IN THE BRAIN}

Vinogradov $\mathrm{SV}^{1}$, Senanayake $\mathrm{T}^{1}, \mathrm{Lu} \mathrm{\textrm {Y } ^ { 1 }}$, Gorantla $\mathrm{S}^{2}$, Poluektova $\mathrm{LY}^{2}$, Makarov $\mathrm{E}^{2}$, Warren $\mathrm{G}^{1}$, Gerson $\mathrm{T}^{1} .{ }^{1} \mathrm{COP}$ Pharmaceutical Science, ${ }^{2}$ Department of Pharmacology and Experimental Neuroscience, University of Nebraska Medical Center, Omaha, NE 68198.

Nucleoside reverse transcriptase inhibitors (NRTI)-formulated nanogels are novel potential drugs for antiretroviral therapy (ART) in the brain. Standard ART is unable to eradicate infection from virus reservoirs in the brain and often induce neurologic complications due to the mitochondrial toxicity of NRTIs. Brain-targeted nano-NRTIs or nanodrug cocktails were evaluated by ability to suppress HIV-1 infection and reduce neuronal damage due to the virus-related inflammation or drug cytotoxicity. Biodegradable cationic nanogels fabricated from PEGylated cholesteryl-polyamines (e.g. epolylysine) and loaded with triphosphorylated Didanosine, Zidovudine, Lamivudine or Abacavir were tested as single nanodrugs, or nanodrug cocktails similar to therapeutic Combivir and Trizivir formulations in infected macrophages. Sustained release of phosphorylated NRTIs does not induce mitochondrial damage. Neuronal apoptosis and drugtriggered production of primary neurotoxic intermediates ROS was visibly reduced after the treatment with nanoNRTIs. Nanodrugs exhibited 5-14-fold higher anti-HIV activity compared to NRTIs and a therapeutic index up to 400 . Additional three to six-fold higher inhibitions were observed 
for dual- and triple-drug nano-NRTI cocktails similar to therapeutic Combivir and Trizivir cocktails, respectively. NanoNRTIs modified with selected brain-specific peptides (e.g. combinatorial brain-binding peptides) demonstrated up to three times better blood-brain barrier (BBB) permeability than nanodrugs in an in vitro cellular BBB model. The excellent accumulation in vivo resulted in the substantial 10-fold inhibition of viral activity in humanized mouse model of HIV1 infection in the brain. Significant suppression of HIVrelated brain inflammation was also observed after 2 weeks of nano-NRTI treatment. In conclusion, nanoformulations of phosphorylated NRTIs represent new promising, effective, and less neurotoxic alternative to current antiviral therapeutics.

047. PHARMACOKINETIC AND IMMUNEDYNAMICS OF MULTI-MODAL NANOPARTICLES FOR HIVAND/OR TB

Reynolds JL, Prasad P, Morse G, Schwartz SA. University at Buffalo, The State University of New York, Buffalo, NY 14202.

HIV is ranked globally as the deadliest single most infectious agent, with TB following a close second. An innovative therapy for these diseases would combine the antimicrobial effects of current drugs with an augmented innate immune system to eradicate these pathogens. These pathogens reside within macrophages and modulate intracellular reactive oxygen and nitrogen species (ROS/RNS) and pro-inflammatory cytokines. We have designed glucan functionalized chitosan shell, poly(lactide)co-glycolide core nanoparticles to stimulate ROS/RNS, pro-inflammatory cytokine secretion, and delivery of rifampicin (TB drug) and/or Nevirapine (HIV drug) inside a 2D-human alveolar like macrophage (ALM) model. Nanoparticles significantly enhanced ALM secretion of IL-12p70 (2.9fold), TNFa; (16-fold) and INFy; (23-fold) compared to controls over $24 \mathrm{~h}$, and doubled ROS/RNS generation over $6 \mathrm{~h}$. Nanoparticles delivered 4-fold greater rifampicin concentrations into ALM compared to free rifampicin solution. We then developed a 3D Hollow Fiber Membrane (HFM) Pharmacokinetic (PK) model to determine the PK of these nanoparticles. This model consisting of an extra-capillary space (ECS) and a central reservoir physically separated by a semi-permeable hollow fiber membrane. We demonstrate that nanoparticles from the central reservoir can transmigrate across the HFM $(0.1 \mu \mathrm{m}$ pore size) to the ECS. We next investigated a time course study in which nanoparticles encapsulated with a fluorescent label (FITC), without drug, were administered in the central reservoir and samples were taken from both the reservoir space and the ECS. Results demonstrate the fluorescence intensity in the reservoir space decreased while the intensity increased in the ECS. These data indicate that these nanoparticles crossed the HFM and validate the potential of this $3 \mathrm{D}$ model for nanoparticle $\mathrm{PK}$ studies. These data demonstrate the feasibility of delivering HIV and/or TB drugs while stimulating the innate immune system.

\section{POSTER ABSTRACTS}

(in alphabetical order)

\section{ANTIANGIOGENESIS BASED NANOTHERAPY FOR PROSTATE CANCER}

Aalinkeel $\mathrm{R}^{1}$, Nair $\mathrm{B}^{1}$, Chen $\mathrm{C}^{2}$, Prasad $\mathrm{PN}^{3}$, Cheng $\mathrm{C}^{2}$, Reynolds $\mathrm{JL}^{1}$, Sykes $\mathrm{DE}^{1}$, Mahajan $\mathrm{SD}^{1,3}$, Chadha $\mathrm{K}^{4}$, Schwartz SA ${ }^{1,3}$. ${ }^{1}$ Department of Medicine, Division of Allergy, Immunology, and Rheumatology, ${ }^{2}$ Department of Chemical and Biological Engineering, ${ }^{3}$ Institute for Lasers, Photonics and Biophotonics, University at Buffalo, The State University of New York 14203, ${ }^{4}$ Department of Molecular and Cellular Biology, Roswell Park Cancer Institute, Buffalo, NY 14263.

Outcome studies of cancer therapy using angiogenesis inhibitors demonstrated substantial clinical benefits. However there is still need for improving their overall efficacy. This can be achieved by facilitating delivery of anti-angiogenesis agents to specific cells using tumor-targeted nanoparticles. In this study we validated a novel approach to inhibit angiogenesis by prostate cancer $(\mathrm{CaP})$ cells using a small interfering (si)RNA specific for interleukin (IL)-8 with a goal to develop a safe, biodegradable nanotherapy for local and metastatic CaP. We used allyl-functionalized L-lactide monomers to yield nanoparticles consisting of a polylactic acid (PLA) backbone. PLA has been previously shown to be biocompatible and biodegradable, making it an ideal clinical agent for nanotherapy. We complexed our PLA nanoparticles with IL-8 specific siRNA yielding a PLA-IL-8-siRNA nanoplex. These nanoplexes were readily taken up by human PC-3 CaP cells resulting in significant silencing of IL-8 gene expression and cell death. In vivo studies were conducted with athymic, nude mice bearing PC-3 tumors. PLA-IL-8-siRNA nanoplexes were injected directly into the tumors. Animals were followed prospectively and showed significant regression of tumors compared to untreated controls. Animals treated with PLAIL-8-siRNA nanoplexes showed significant knockdown of IL-8 gene expression in tumor tissues and decreased levels of angiogenic and anti-apoptotic cytokines (FTF2, follistatin, GCSF, IL-17A, and IL-6) in serum compared with untreated controls. Further, imaging of tumors showed reduced volume and vascularization by PLAIL-8-siRNA nanoplex treated tumors both at 79 and 101 days post tumor inoculation consistent with diminished angiogenesis. Our study establishes proof of concept for using nanoparticles to deliver gene therapy for the treatment of $\mathrm{CaP}$. Our next studies will use targeted nanoplexes to treat metastatic human $\mathrm{CaP}$ in an animal model prior to commencing clinical trials 


\section{NANOAPPROACH FOR EFFECTIVE BRAIN OF NATURAL ANTIEPILEPTIC DRUG}

Akhter S. Department of Pharmaceutics Utrecht Institute for Pharmaceutical Sciences (UIPS) Utrecht University, Universiteitsweg 99. 3584 CG.

Thymoquinone (THQ) is known for its neuroprotective and anti-convulsant properties in preclinical studies. In this work describes the improved brain targeting of Thymoquinone (THQ) via intranasal route using mucoadhesive nanoemulsion as carrier. THQ loaded mucoadhesive nanoemulsion was prepared by aqueous titration technique and in-vitro evaluation for stability and release. Anti-convulsant efficacy, brain pharmacokinetics, biodistribution and safety of the THQ nanoformulation was performed in rabbits model.

050. HYPERINTENSE CORTICAL T1 SIGNAL IN PROGRESSIVE LEUKOENCEPHALOPATHY (PML) REFLECTS SEIZURE RISK

Anwar B. University of Oran, Algeria.

Objective: To determine the frequency of hyperintense cortical T1 signal (HCTS) on MRI in progressive leukoencephalopathy (PML) patients, its association with seizure risk and immune reconstitution inflammatory syndrome (IRIS). Background: PML, caused by JC virus (JCV) affects predominantly white matter.seizures point to a cortical origin and add to increasing evidence of gray matter involvement in PML. HCTS is a radiologic marker of cortical laminar necrosis. Methods: We reviewed clinical data including seizure history, presence of IRIS, and MRI scans from PMl patients evaluated between 2000 and 2012. Cases that were diagnosed by CSF JCV PCR and who had MRI images available were included in the analysis $(n=45)$. Results: Of 45 patients, 16 (35\%) had seizures and 27(61\%) had HCTS on MRI. Of the 16 PML patients with seizures, 14 (88 \%) had HCTS compared to 13/29(44\%) patients without seizures $(p=0.006)$. Of the 18 patients with IRIS, $14(80 \%)$ had HCTS compared to $13 / 27(49 \%)$ to those without IRIS, $(p=0.04)$.HCTS predicted seizures with an odds ratio(OR) of $8.5(95 \%$ confidence interval(CI) of $1.66-$ 43.41). Together HCTS and IRIS predicted seizures with an Or of $9.9(95 \%$ CI of $2.5-39.3 ; p=0.001)$.Conclusion: Seizures are a frequent complication in PML .HCTS is associated with seizures as well as IRIS.

\section{MEMRI REFLECTS HUMAN NEUROPATHOLO- GY IN MURINE HAND}

Bade $\mathrm{A}^{1}$, Gorantla $\mathrm{S}^{1}$, Dash $\mathrm{P}^{1}$, Makarov $\mathrm{E}^{1}$, Poluektova $\mathrm{LY}^{1}$, Gendelman $\mathrm{HE}^{1}$, Boska $\mathrm{MD}^{2}$, Liu $\mathrm{Y}^{2}$. ${ }^{1}$ Department of Pharmacology and Experimental Neuroscience and ${ }^{2}$ Department of Radiology, University of Nebraska Medical Center, Omaha, NE 68198.
Progressive HIV infection commonly leads to cognitive impairments for HIV associated neurocognitive disorders. Our labs have successfully reproduced the biology, immunology and virology of HAND in mice. This is timely as, no reliable diagnostic test for human disease is available and disease tracking and therapeutic monitoring are not available in animal models. We believe we have found an answer to this bottleneck. To this end, we recently developed a manganese enhanced MRI (MEMRI) method to reflect central nervous system damage following HIV infection. This is based on the abilities of Mn2+ acting as a calcium analog where it enters into neurons through calcium channels. Thus, the $\mathrm{T} 1$ relaxivity changes, and hence $\mathrm{T} 1$ weighted signal intensity, are quantitatively proportional to calcium channel $\mathrm{Mn} 2+$ activities. MEMRI was used to examine brains of humanized mice infected by HIV-1. Changes in MEMRI signal enhancements during HIV-1 infection showed profound changes in $\mathrm{Mn} 2+$ accumulation suggesting associated neural pathology. This was observed in disease affected brain regions. Multiple linear regression analysis showed changed MEMRI signal enhancements positively correlated with high viral loads at study end $(r=0.78 p<0.05)$ and negatively with peripheral CD4+ T cells $(r=-0.437 p<0.05)$. Quantitative immunohistological results showed that GFAP reactions correlated positively with signal enhancements in CA1 $(r=0.96$, $p<0.05)$, CA3 $(r=0.89, p<0.5)$ and the dentate gyrus $(r=$ $0.95, p<0.05)$. This was negatively correlated with synaptophysin in the CA3 $(r=-0.98 p=<0.05)$ and the sensory regions of cortex $(r=-0.87, p=0.05)$. The abnormal signal intensity correlated with viral pathology in infected mice supporting the emergence of behavioral deficits in infected animals. We conclude MEMRI could be used to investigate specific neuropathologic aberrations associated with advanced viral infection and can be used to monitor therapeutic efficacy to combat neuronal damage in HAND.

Supported by: NIH P01DA028555, R01NS036126, R01NS34239 (HEG)

\section{NEUROPROTECTIVE EFFECT OF BANANA FLOWER ON A LIPOPOLYSACCHARIDE-INDUCED ANIMAL MODEL OF PARKINSON'S DISEASE}

Chen W. Chung Yuan Christian University, Chung Li, Taiwan, Republic of China.

Lipopolysaccharide (LPS) induces microglial activation to release a variety of neurotoxic factors, and damaged neurons may trigger reactive microgliosis, which lead to progressive dopaminergic neurodegeneration. It has been used to study the neuroinflammatory process in the pathogenesis of Parkinson's disease (PD) and anti-inflammatory therapy for PD treatment. Here, the unilateral stereotaxic injections of LPS into the substantia nigra of male rats are performed to establish the hemiparkinsonian animal model. Banana flower (Musa Sapientum) has been shown to exhibit antioxidant activity 
and anti-diabetic property. However, the effect of banana flower treatment on neurodegeneration has not been evaluated. The objective of this study was to evaluate the neuroprotection of banana flower in LPS PD model. Rotation behavioral studies showed banana flower attenuated the associated motor deficits, prevented neurodegeneration. Further observation revealed banana flower pre-treatment significantly ameliorated LPSinduced elevations of the mRNA expressions of interleukin-1 beta (IL-1 $\beta$ ). Our results suggest that banana flower may play a role in protecting dopaminergic neurons against inflammatory challenge.

053. EVALUATION OF LONG TERM TOXICITY OF GRAFEX NANOCARBON ASSISTED MICROWAVE THERAPY (NAMT) FOR APPLICATION OF TARGETING HUMAN PROSTATE TUMOR CELLS IN NUDE MICE CAUSING CYTOTOXIC THERMAL ABLATION USING EXTREMELY SHORT CYCLE MICROWAVE ENERGY AS PRIMARY THERAPY

DeSantis M. State University of New York at Stony Brook, Stony Brook, NY 11790.

Purpose: Evaluation of long term toxicity using application of spherical nanocarbon injected into known Human Prostatic carcinoma to increase absorption of microwave energy, specifically into tumor cells. This study was selected to evaluate NAMT can be used as primary treatment and evaluate the toxic burden of nanocarbon used during treatment. This specific nanocarbon has demonstrated a range of energy absorption capabilities. Materials and Methods: 10 Nude nu/nu isolated mice were injected with DU145 (ATC\#HTB-81) $1 \times$ $10^{\wedge} 7$ Human Prostate carcinoma cells introduced into the dermis and allowed to grow to $>1 \mathrm{~cm}$. A control mouse received no treatment. A control mouse received only an injection of 292 molar concentration of nanocarbon. Remaining 8 mice received treatment with the microwave and 292 molar nanocarbon. Medwave generators with microwave probes were used. Nanocarbon and viscous carrier were injected into the tumors. Short cycle power using 10 watts at $15 \mathrm{~s}$ used as baseline settings. Target temperature within the tumor was $60^{\circ} \mathrm{C}$. Results: $67 \%$ of the original treated mice are alive at 18 months with no signs of toxicity or tumor re growth. Conclusion: Grafex nanocarbon appears to be nontoxic in this small animal study. NAMT maximizes energy transfer. Conversion of microwave energy causes thermal ablation to the cancer cells. This suggests Grafex nanocarbon causes enhancement of the dielectric properties of the tumor causing cytotoxic heating. A larger study is under way.

054. DELIVERY OF ANTITUBERCLIN DRUG NANOPARTICLES TO SPECIFIC MACROPHAGE SUBCELLULAR COMPARTMENTS FACILITATES ELIMINATION OF MYCOBACTERIAL INFECTION
Edagwa B, Guo D, Puligujja P, McMillan J, Liu X, Gendelman HE, Narayanasamy P. University of Nebraska Medical Center, Omaha, NE 68198.

Mycobacterium tuberculosis (MTB) is the cause of widespread pulmonary, bone, nervous system, skin, eye and other systemic morbidities. Complex drug regimens underlie an alarming worldwide increase in infection rates. The needs for new treatment regimens to improve patient compliance and speed microbial eradication are surely needed. We posit that this may be achieved through long-acting, cell and tissue targeted, nanoformulated MTB medicines. To this end, we developed a derivatized hydrophobic isoniazid now called INHP. INHP and rifampin (RIF) were loaded into polylactide-coglycolic acid (PLGA) nanoparticles. Physical and chemical properties of the drug formulations as well as the resultant particle cell uptake, retention, viability and antimicrobial efficacy were determined in relationship to native drugs. These nanoformulations displayed enhanced uptake of 6 and $4 \mu \mathrm{g}$ of drug/106 cells when compared to $0.1 \mu \mathrm{g} /$ 106 cells for native drugs. Sustained release of encapsulated INHP and RIF were detectable in monocyte-derived macrophages (MDM) over 15 days whereas native drug was released within $24 \mathrm{~h}$. Antimicrobial activities as determined in dose response tests against Mycobacterium smegmatis showed $\sim 6$-fold increases over equivalent concentration of native drug when both INHP and RIF nanoformulations were administered together. Endocytic trafficking of the antimycobacterium nanoparticles and $\mathrm{M}$. smegmatis showed identical subcellular compartment localization in recycling and late endosomes. In conclusion, PLGA nanoparticles encapsulating RIF and INHP significantly improves drug uptake and retention and antimicrobial activities from native drugs. Subcellular distribution study of M. smegmatis and the PLGA nanoformulations show that the drugs and the mycobacterium are trafficked into equivalent endocytic compartments. These results highlight new opportunities for antimicrobial nanomedicines to simplify and improve the means to affect MTB eradication.

Supported by: NIH P01DA028555 and R01 A1097550 (HEG)

\section{NANOFORMULATED ART ENDOCYTIC TRAFFICKING FACILITATES HUMAN MONOCYTE- MACROPHAGE PARTICLE CARRIAGE}

Guo $D^{1}$, Zhang $\mathrm{G}^{1}$, McMillan $\mathrm{JM}^{1}$, Liu $\mathrm{X}^{1}$, Wysocki $\mathrm{BJ}^{2}$, Wysocki T ${ }^{2}$, Gendelman $\mathrm{HE}^{1} .{ }^{1}$ Department of Pharmacology and Experimental Neuroscience, University of Nebraska Medical Center, Omaha, NE 68198 and ${ }^{2}$ Computer and Electronics Engineering Department (CEEN Dept.), Wireless Research Laboratory, University of NebraskaLincoln, Lincoln, NE 68182. 
Background: Long-acting nanoformulated antiretroviral therapy (nanoART) can improve patient drug adherence, decrease systemic toxicity and affect sustained viral suppression. This occurs through its abilities to maintain consistent plasma and tissue drug levels. Here, monocyte-macrophages serve as ART carriers. Specific intracellular compartments traffic nanoART and promote slow drug release. Methods Nanoatazanavir(nanoATV) was prepared by high-pressure homogenization with poloxamer 407. Endosomal compartments were isolated from nanoATV treated human monocyte-derived macrophages (MDM) from 0.5 to $48 \mathrm{~h}$ then purified by immunoaffinity chromatography with Rabspecific antibodies conjugated to magnetic beads. Confocal microscopy confirmed subcellular nanoATV distribution. NanoATV distribution into early, recycling and late endosome/lysosomal fractions were determined by immunofluorescence staining. Mathematical models were developed by computer assistance programming simulated particle trafficking activities. Results Particle size and zeta-potential was optimized for particle MDM uptake and storage assays. Particles were efficiently engulfed by MDM at $53.5 \mathrm{ug} / 106$ MDM after $16 \mathrm{~h}$, which was 1,000 times higher than freebased drug. Subcellular compartments (Rab 5, 7, 11 and 14) were separated and the nanoATV concentrations were 5.5, 6.5, 8.3 and $10.6 \mathrm{ug} / 106 \mathrm{MDM}$, respectively. With computer assistance, the nanoATV uptake activity was simulated in endocytic compartments. Confocal imaging showed particle distribution in a punctate pattern in cytoplasmic and perinuclear regions. Antiretroviral studies showed reduction in reverse transcriptase activity by 93 and $77 \%$ with nanoATV and free-based drug on challenge $14 \mathrm{~d}$ for late endosomes. Conclusions: The subcellular locale of the nanoATV and its slow release underlie the developments of long-term antiretroviral efficacy. Macrophages act as drug transporters keeping ART nanoparticles stable in late endosomes for prolonged time periods.

Supported by: NIH P01DA028555, R01NS036126, R01NS34239 (HEG)

056. CATIONIC/AMPHIPHILIC PEPTIDE/NUCLEIC ACID COMPLEXES SPECIFICALLY TRAVERSE CANCER CELL MEMBRANES AND RELEASE THEIR FUNCTIONAL NUCLEIC ACID CARGO

Gupta $\mathrm{K}^{1}$, Afonin $\mathrm{K}^{1}$, Viard $\mathrm{M}^{1}$, Puri $\mathrm{A}^{1}$, Zakharov $\mathrm{A}^{2}$, Nicklaus $\mathrm{MC}^{2}$, Schneider $\mathrm{J}^{3}$, Shapiro BA ${ }^{1}$. ${ }^{1}$ Basic Research Laboratory, ${ }^{2}$ Computer-Aided Drug Design, Chemical Biology Laboratory, ${ }^{3}$ Peptide Design and Materials Section, Chemical Biology Laboratory, National Cancer Institute, National Institutes of Health, Frederick, MD 21701.

Among non-viral vectors, cell penetrating peptides (CPPs), which generally contain a stretch of cationic and/or amphipathic peptides of 5-30 amino acids, have received considerable attention for their ability to transport nucleic acids into cells both in vitro and in vivo. Recent reports revealed that cancer cells are anionic due to the overexpression of negatively charged phosphatidylserine (PS) lipid and high levels of sialic acid in comparison to normal cells. These characteristics can be exploited to improve delivery of therapeutic nucleic acid candidates. Herein we describe the rational design and synthesis of cationic/amphiphilic peptides that upon complexing with nucleic acids form beta-hairpins and impart amphiphilicity to the peptide/nucleic acid complex. This facilitates the specific traversal of anionic cancer cell membranes and enhances the delivery of a variety of nucleic acid structures that control cellular functions. We have designed a series of peptides containing alternating hydrophobic and polar/positively charged residues connected by a type II' turn promoting sequence $\mathrm{V}^{\mathrm{D}}$ PPT that forms beta-hairpins and tested them for transfection and for Green Fluorescent Protein (GFP) gene silencing efficiency in human breast cancer cells (MDAMB231). The ability of these peptides to form beta-hairpins upon complexing with nucleic acids was verified by circular dichrosim spectroscopy with MAX35 peptides, which showed beta-hairpin formation in the presence of siRNAs. As an example, the HPL24 peptides showed significant transfection of fluorescently labeled RNA/DNA duplexes and were capable of delivering siRNA to efficiently silence GFP expression in the breast cancer cells as revealed by Fluorescence activated cell sorting (FACS) analysis. However, MAX8V16E, designed to be a non beta-hairpin forming control peptide, did not show significant transfection. Also, QSAR was able to predict some of the characteristics of these peptides.

\section{DELIVERY OF GDNF TO THE BRAIN BY NOVEL NANOVESICLES FOR THE TREATMENT OF PARKINSON'S DISEASE}

Heldman $\mathrm{E}^{1,2}$, Grinberg $\mathrm{S}^{1,2}$, Linder $\mathrm{C}^{1,2}$, Popov $\mathrm{M}^{1,2}$, Hollander $\mathrm{I}^{1,2}$. (presenter: Rosenbaum $\left.\mathrm{S}^{1}\right)^{1}$ Lauren Sciences LLC, NY and ${ }^{2}$ Ben-Gurion University of the Negev, Israel.

Neurotrophins like GDNF have good potential as therapeutic agents in Parkinson's (PD). However, neurotrophins do not penetrate the $\mathrm{BBB}$ and a targeted delivery system is needed to derive their therapeutic potential. Here we describe optimized VSmartTM vesicles (based on our nanovesicles shown to cross the BBB and deliver proteins and siRNA) capable of delivering GDNF to the striatum and the substantia nigra - brain regions affected in PD. Nanovesicles were formed from synthetic bolaamphiphiles containing: acetylcholine head groups that enable release from the vesicles upon exposure to $\mathrm{AChE}$; targeting ligand for targeting to dopaminergic neurons; and chitosan moieties that enable penetration through the BBB. Carboxyfluorescein-loaded vesicles were added to cultured cells, then studied by FACS. GDNF-loaded vesicles were administered intravenously to mice, which were then perfused 
with PBS at various times after vesicle administration, brains removed, homogenized or sectioned for histology, and brain GDNF determined quantitatively by ELISA and localized by histofluorescence. Vesicles decorated with targeting ligand bind to cultured cells that express receptor significantly more than naked vesicles do, whereas no difference is found between naked and decorated vesicles in cultured cells that do not express receptor, suggesting that in vivo specific targeting to dopaminergic cells would occur. GDNF, encapsulated in vesicles decorated with targeting ligand, was administered intravenously to mice and found in significant amounts in the brain with preferential localization in the striatum and substantia nigra - brain regions rich in dopaminergic cells. V-SmartTM vesicles, optimized for targeted delivery of GDNF, successfully delivered GDNF into areas of the brain affected by Parkinson's and, thus, show great potential for PD therapy. Results of preliminary efficacy studies in PD animal models will also be presented. Supported by: The Michael J. Fox Foundation

\section{DELIVERY OF TENOFOVIR TO THE BRAIN BY NOVEL NANO-VESICLES FOR THE TREATMENT OF NEURO-HIV}

Heldman $\mathrm{E}^{1,2}$, Grinberg $\mathrm{S}^{1,2}$, Linder $\mathrm{C}^{1,2}$, Popov $\mathrm{M}^{1,2}$, Hollander $\mathrm{I}^{1,2}$. (presenter: Rosenbaum $\mathrm{S}^{1}$ ) ${ }^{1}$ Lauren Sciences LLC, NY and ${ }^{2}$ Ben-Gurion University of the Negev, Israel.

Many AIDS patients, on Highly Active Anti-Retroviral Treatment (HAART), have non-detectable HIV in the blood and, yet, measurable amounts in the CNS because of poor BBB penetrability of some HAART drugs, particularly tenofovir. Persistent HIV reservoirs in the CNS are considered responsible for neurological impairment in AIDS patients. We have synthesized novel bolaamphiphiles that form stable monolayer-membrane vesicles, characterized by high encapsulation efficiency, penetrability through the BBB and efficient release of the encapsulated material within the brain. Here we describe optimized V-SmartTM vesicles capable of delivering tenofovir into the brain to make HAART treatment more effective. Nanovesicles were formed from various formulations that contain our novel bolaamphiphiles (bolas) and tenofovir. Tenofovir encapsulation was determined following the removal of non-encapsulated tenofovir by size-exclusion chromatography. The vesicle formulation was optimized to achieve high tenofovir encapsulation and good BBB permeability. Optimized vesicles were administered to mice and at various times after vesicle administration, brain tenofovir levels were determined by LC/MS. Optimal vesicle formulations produced $20-40 \%$ tenofovir encapsulation. Incorporation of new bolas with chitosan head groups to the formulation increased significantly the penetration of the vesicles to the brain. A single injection (iv) of tenofovir-loaded vesicles (doses 10-fold below toxic levels) resulted in brain tenofovir concentration of $\sim 6 \mathrm{ug} / \mathrm{gm}$ tissue, much higher than the therapeutic levels of $\sim 100 \mathrm{ng} / \mathrm{gm}$, which is a typical blood level in HAART patients.

Thus, V-SmartTM vesicles, optimized for brain delivery of tenofovir, successfully delivered therapeutic amounts of the drug into the brain after a single iv administration. The results indicate the feasibility of using V-SmartTM vesicles for lowering viral load in the CNS of HIV patients.

Supported by: The Campbell Foundation

\section{CREATION OF SUSTAINED RELEASE MARAVIROC NANOPARTICLES}

Hilaire J, Puligujja P, McMillan J, Liu X, Gendelman HE. Department of Pharmacology and Experimental Neuroscience, University of Nebraska Medical Center, Omaha, NE 68198 .

Maraviroc (MVC; SELZENTRY) is a well-known CCR5 coreceptor antagonist commonly used as part of combination antiretroviral therapy (cART). A twice-daily dosing regimen, limitations in virologic suppressive activity, as well as lack of specific delivery to sites of viral entry, limits its maximal efficacy. On balance, when used with select antiretrovirals its efficacy is significant and sustained. We sought to overcome MVC limitations by packaging the drug into nanoparticles enabling monocyte-macrophage uptake and delivery. The goal is to create a cell-based drug depot, which will facilitate particle dissolution and subsequent sustained release of MVC at its action site. To this end, polymeric lipid-coated (PLGA)-MVC nanoparticles were manufactured. Physicochemical characteristics of nanoformulated MVC, as well as human monocyte-derived macrophage (MDM) uptake, retention, release and antiretroviral responses were examined. MDM treated with nanoformulated MVC (nMVC) or native MVC for $8 \mathrm{~h}$ exhibited intracellular drug levels of 10.7 and $3.9 \mathrm{ug} / 106$ cells, respectively. Ten days following nMVC treatment $(100 \mu \mathrm{M})$ MDM retained 1.06 ug MVC/106 cells while native MVC was undetectable. Antiretroviral activity was determined by reverse transcriptase (RT) activity and HIV-1p24 antigen expression in HIV-1 infected MDM. MDM challenged with HIV$1 \mathrm{ADA}>15$ days after $8 \mathrm{~h}$ nMVC treatment exhibited dose dependent reductions in RT activity. At a concentration of $100 \mathrm{uM}, \mathrm{nMVC}$ and native MVC resulted in 95 and $40 \%$ RT activity reductions compared to untreated infected cells respectively. These results demonstrate that nMVC creates a cellular drug depot, which facilitates effective antiretroviral efficacy weeks after treatment. To further characterize nMVC, we are determining the PK profile. Overall, development of long acting targeted MVC together with reverse transcriptase, protease and integrase inhibitors could lead to improved PK of cART and promote improved drug adherence. 
Supported by: NIH P01DA028555, R01NS036126, R01NS34239 (HEG)

\section{ENDOCYTOSIS PATHWAYS OF THE FOLATE TETHERED STAR-SHAPED PEG-PCL MICELLES IN CANCER CELL LINES}

Hsieh M-F. Department of Biomedical Engineering, Chung Yuan Christian University, Taiwan 32023, R.O.C.

This study reports on the cellular uptake of folate tethered micelles using a branched skeleton of poly(ethylene glycol) and poly(\&\#949;-caprolactone). The chemical structures of the copolymers were characterized by proton nuclear magnetic resonance spectroscopy, and Fourier transform infrared spectroscopy. Doxorubicin (DOX) was utilized as an anticancer drug. The highest drug loading efficiencies of DOX in the folate decorated micelle (DMCF) and folate-free micelle (DMC) were found to be $88.5 \%$ and $88.2 \%$, respectively, depending on the segment length of the poly(\&\#949;caprolactone) in the copolymers. A comparison of fluorescent microscopic images of the endocytosis pathway in two cells lines, human breast cancer cells (MCF-7) and human oral cavity carcinoma cells (KB), revealed that the micelles were engulfed by KB and MCF-7 cells following in vitro incubation for $1 \mathrm{~h}$. Flow cytometric analysis revealed that free folic acid can inhibit the uptake of DOX loaded in the micelles by $48-57 \%$ and $26-39 \%$ in KB cells and MCF-7 cells, respectively. These results prove that $\mathrm{KB}$ cells are relatively sensitive to folatetethered micelles. Upon the administering of methylbeta-cyclodextrin, an inhibitor of the caveolae-mediated endocytosis pathway, the uptake of DOX by $\mathrm{KB}$ cells was reduced by $69 \%$ and that by MCF-7 cells was reduced by $56 \%$. This finding suggests that DMCF enters cells via multiple pathways, so folate receptor is not the only target of tumor therapeutics.

\section{HAP AND GENOTOXICITY BUDDING TOWARDS NANOMEDICINE}

Huang $\mathrm{Y}^{1,2}$, Yamauchi $\mathrm{Y}^{3,4,5}$, Rawat $\mathrm{A}^{1}$. ${ }^{1}$ Department of Bioenvironmental Engineering and Research Center for Analysis and Identification, ${ }^{2}$ Center of Biomedical Technology (CBT), Chung Yuan Christian University, No. 200, Chung-Pei Road, Jhong-Li 32023, Taiwan; ${ }^{3}$ World Premier International Research Center for Materials Nanoarchitectonics, National Institute for Materials Science (NIMS), 1-1 Namiki, Tsukuba, Ibaraki 305-0044, Japan; ${ }^{4}$ Faculty of Science and Engineering, Waseda University, 3-4-1 Okubo, Shinjuku, Tokyo 169-8555, Japan; ${ }^{5}$ PRESTO, Japan Science and Technology Agency (JST), 4-1-8, Honcho, Kawaguchi, Saitama 332-0012, Japan.

Engineered silver nanomaterials have been touted as panacea in nanomedicine sector. Nanocomposite materials, such as
Hydroxyapatite (HAP: Ca10(PO4)6(OH)2) and the nanohydroxyapatite particles ensures biocompatibility, and used for dental fillings, implant coatings and bone substitutes. The reaction mechanism between these material and microorganism still remains obscure; we attempted to perceive it through a stable reference gene for evaluating the antibacterial activity of HAP. The minimum inhibitory concentration (MIC) and half maximal inhibitory concentration (IC50) tests were conducted with Escherichia coli strain TOP10 treated metal-coated HAP. The results manifest that the biomaterial has high surface area and uniform size distribution. The inclusion of metal with HAP, like gold and silver imparted them greater toxicity, than regular non-coated ones. MIC test confirmed that silver was more toxic than gold. To get an insight of mechanism, we scrutinized six genes namely, DNA polymerase I (polA), DNA polymerase II (polB), cytochrome d complex (cyd), glucan biosynthesis protein $\mathrm{G}$ (mdoG), D-glyceraldehyde-3-phosphate dehydrogenase (GAPDH), and 16S ribosomal RNA (16S rRNA), and performed quantitative reverse transcription polymerase chain reaction (qRT-PCR) for a complete gene expression analysis. The expressions of cyd and mdoG were inhibited, illustrating that the antibacterial activity of HANPs is related to cell membrane or cell wall proteins. This study explicitly shows the mechanism of cellular organism with HAP. Attributes like biocompatibility and genotoxicity can serve as a reference in synthesis of novel nanocomposite biomaterials or potentially biomedicines. Nanomedicines currently hold a tiny niche in pharmaceutical industry, but recent accomplishments in genetic level has certainly opened doors for this stream to excel.

\section{NANOCONJUGATES OF AGONISTS AND ANTA GONISTS OF G PROTEIN-COUPLED RECEPTORS POTENT MULTIVALENT LIGANDS OF ADENOSINE AND P2Y RECEPTORS}

Jacobson KA, Tosh, DK, Jayasekara, PS. Laboratory of Bioorganic Chemistry \& Molecular Recognition Section National Institute of Diabetes \& Digestive \& Kidney Diseases, National Institutes of Health, Bethesda, MD 20892.

Extracellular nucleosides and nucleotides acting at $\mathrm{G}$ proteincoupled receptors (GPCRs) are important biological signaling molecules. They are of interest as pharmacological probes and potential therapeutic agents, including anticancer drugs. With detailed knowledge of structure activity relationships at both adenosine receptors (ARs) and $\mathrm{P} 2 \mathrm{Y}$ receptors for nucleotides, we designed selective functionalized ligands for synthesis of multivalent bioactive nanoconjugates, including soluble PAMAM dendrimer conjugates and solid nanoparticles (NPs). PAMAM conjugates of A3AR agonists are efficacious in cardioprotection models. AuNPs allow the tuning 
of pharmacokinetic and pharmacodynamic properties by the active or passive targeting of drugs for cancer and other diseases. We have functionalized AuNPs by tethering specific ligands, nucleoside agonists and xanthine antagonists, of ARs as models for cell surface interactions with GPCRs. These strategically-derivatized NPs, especially agonists of the anti-inflammatory A3AR, retained high binding affinity as the covalent conjugates. This is the first prototypical application to gold carriers of small molecule (nonpeptide) GPCR ligands, including agents under investigation for the treatment of cancer and inflammatory diseases. Strategies for immobilizing A2AAR agonists (anti-inflammatory activity) have been explored in nucleoside conjugates of quantum dots for receptor characterization. The approach of tethering properly functionalized GPCR ligands to nanocarriers promises to provide potent new materials for biomedical applications.

\section{ENCAPSULATION OF NEEM NANOEMULSION FOR CONTROLLING URINARY TRACT INFECTION CAUSING BACTERIA KLEBSIELLA PNEUMONIAE Jerobin J, Singh M, Sahni RD, Mukherjee A, Chandrasekaran N. VIT University, Vellore, India.}

Urinary tract infection (UTI) is a common problem in women than men which affects the urinary tract of human. The Klebsiella pneumoniae is a common bacterium which causes urinary tract infection. Most of the antibiotics currently available are found to be resistance against the UTI causing bacteria, and a need has arisen for new antibacterial agent. The nanoemulsion is kinetically stable non toxic lipid droplet formulated using pharmaceutical ingredients suitable for human consumption. The neem (Azadirachta indica) is a medicinal plant which has antibacterial, antifungal, antiviral and antimalarial activities. The oil in water neem nanoemulsion is formulated using neem oil, suitable surfactant and Milli Q water at different ratio. The 1:3 ratio of neem nanoemulsion is found to be stable with a hydrodynamic size of $31 \mathrm{~nm}$. The neem nanoemulsion is almost spherical in shape when examined by TEM. The formulated and characterized neem nanoemulsion was encapsulated with suitable polymer. The FTIR and the controlled release of azadirachtin a biological compound in neem confirmed the encapsulation of neem nanoemulsion. The PEG coated encapsulation of neem nanoemulsion was found to be effective against the UTI causing bacteria K.pneumoniae (MTCC No: 432) by altering the morphology of the bacteria in vitro. The neem nanoemulsion can be used as an effective antibacterial agent. More in vivo studies have to be done regarding the antibacterial activity of neem nanoemulsion against the UTI causing bacteria.
064. A NANOCOMPLEX CROSSES THE BLOODBRAIN BARRIER AND DELIVERS TO DEEP BRAIN FOLLOWING SYSTEMIC ADMINISTRATION

Kim S-S ${ }^{1}$, Garrido-Sanabria ER ${ }^{2}$, Rait A ${ }^{1}$, Pirollo $\mathrm{KF}^{1}$, Chang $\mathrm{EH}^{1,3} .{ }^{1}$ Department of Oncology, Lombardi Comprehensive Cancer Center, Georgetown University Medical Center, Washington, DC 20057, ${ }^{2}$ Department of Biomedicine, University of Texas Brownsville, Brownsville, TX 78520, ${ }^{3}$ SynerGene Therapeutics, Inc., Potomac, MD 20854.

Despite major breakthroughs in molecular pathogenesis and CNS drug discoveries, the inability of free drugs to efficiently cross the blood-brain barrier (BBB) has been a major impediment in the treatment of neurological disorders including brain tumors. Thus, there is a critical need to develop new technologies to meet the challenge. We have developed a targeted nanodelivery system (scL) for CNS drug delivery wherein the payload is encapsulated within a cationic liposome. The surface of the liposome is decorated with an antitransferrin receptor (TfR) single-chain antibody fragment (TfRscFv) as a targeting moiety. This rational design allows the systemically administered scL nanocomplex to take advantage of the elevated TfR levels present on the cerebral endothelium of the BBB in order to facilitate its transcytosis across the BBB. In vivo studies were performed to assess delivery to the brain of scL after intravenous (tail vein) injection. The scL nanocomplex was shown to deliver a fluorescence-labeled oligonucleotide (Cy5-ODN) to the deep brain, resulting in an accumulation of strong fluorescence signal in specific regions of the mouse brain including hippocampus and cerebellum. Furthermore, tail vein injection of the scL nanocomplex carrying plasmid DNA encoding the EGFP protein also resulted in specific expression of exogenous EGFP in neuronal cells in the deep brain. This scL is a platform technology, and this nanocomplex has been shown to be capable of systemically delivering various payloads including nucleic acid-based therapeutics (plasmid DNA, si/miRNA, antisense ODN), diagnostic imaging probes, small molecules and chemotherapeutic agents to the target tissues. Thus, scL nanodelivery system provides an efficient and less invasive approach for the delivery of therapeutic and diagnostic molecules across the BBB. We believe that this approach has a significant potential for impacting future treatment of CNS disorders.

065. TUNABLE RESISTIVE PULSE SENSING HIGH RESOLUTION SIZE, CHARGE AND CONCENTRA TION MEASUREMENTS FOR TAKING NANOMEDICINES FROM THE LAB TO THE CLINIC Kozak $\mathrm{D}^{1}$, Anderson $\mathrm{W}^{2}$, Vogel $\mathrm{R}^{3}$, Broom $\mathrm{MF}^{3}$. ${ }^{1}$ Izon Science US Ltd, Cambridge, MA, ${ }^{2}$ The University of Queensland, Brisbane, QLD, Australia. ${ }^{3}$ Izon Science Ltd, Christchurch, New Zealand. 
Accurate high resolution characterization of complex systems such as drug delivery particles, viruses, and microvesicles is critical to understanding their function and optimization in diagnostic and therapeutic applications. Tunable resistive pulse sensors (TRPS), such as the Izon qNano and qViro-X, have generated considerable interest for their ability to accurately characterize the size, charge and concentration of nano to micro-scale particulate suspensions. Measuring the properties of each particle (e.g. liposome, exosome, virus, microvesicle, or cell) as is passes through the elastic pore sensor provides high resolution analysis often beyond that of other analysis techniques. Furthermore, the recently developed capability to simultaneously measure the size and charge on a particleby-particle basis provides a unique method to better characterize and understand the role that these properties play. We present the fundamental principles behind TRPS and demonstrate how it has been used to improve the characterization of particle size, charge and concentration within complex (e.g. polydisperse or multimodal size and charge) suspensions. The ability to easily resolve similar but different sized particle populations has been demonstrated on biological and model complex samples composed of particles with diameters of 220,330 and $410 \mathrm{~nm}$. Tuning the sensor gave rise to a 6 fold improvement in the measurement sensitivity and when compared to four other size analysis techniques, TRPS was one of two devices capable of accurately resolving particles within complex samples. These instruments have been used to characterize a wide range of synthetic and biological particle systems including adenovirus, exosomes, DNA coated particles, Baculovirus occlusion bodies, and Prochlorococcus as well as study the surface modification, aggregation, and storage effects on liposomes.

\section{A FOLATE USPIO MRI SCREEN FOR PHARMA- COKINETIC ASSESSMENTS}

$\mathrm{Li} \mathrm{T}^{1}$, Gendelman HE ${ }^{1}$, McMillan JM ${ }^{1}$, Liu X ${ }^{1}$, Boska MD ${ }^{1,2}$. ${ }^{1}$ Department of Pharmacology and Experimental Neuroscience and ${ }^{2}$ Department of Radiology University of Nebraska Medical Center, Omaha, NE, 68198.

Background: Alendronate (ALN) is a bisphosphonate derivative with strong metal-chelating properties. In this report, we successfully harnessed ALN-conjugated polymers and coated them onto ultra-small superparamagnetic iron oxide (USPIO) particles (APM). This served to generate a hydrophilic magnetite particle of $<20 \mathrm{~nm}$. A folate-ALN polymer (FAP) was synthesized to make FAP coated USPIO particles (FAPM) in order to facilitate macrophage targeting for cell-guided tissue particle delivery. Our overarching goal is to use FAPM to investigate targeted antiretroviral nanoparticle (nanoART) delivery to sites of human immunodeficiency virus (HIV) infection. Methods: ALN-conjugated methoxy poly (ethylene oxide), ALN-PEO, was synthesized by click chemistry. The stability of the APM was evaluated with divergent buffers $\mathrm{pH}$ $(2-12)$ and $\mathrm{NaCl}$ salts $(0-2 \mathrm{M})$. The magnetic resonance (MR) T2-weighted relaxivity was determined. Results: ALNPEO coating onto USPIO is rapid, efficient, stable and tunable. Up to $100 \%$ of the polymer coated onto USPIO was seen at a 10 to 1 weight ratio of ALN-PEO/USPIO. The coating was stable in $\mathrm{pH} 2$ to 12 buffers and up to $2 \mathrm{M} \mathrm{NaCl}$ salt solutions. There was no significant increase in size and polydispersity of USPIO in buffer and $\mathrm{NaCl}$ solutions over 1 month of storage. MR relaxivity studies showed that APM has the similar T2-weighted relaxivity constant to that of Ferumoxytol ( 81.50 versus 83.92 , s-1 mM-1). In animals stimulated with lipopolysaccharide, FAPM showed more accumulation in various tissues compared to nontargeted particles $(0.25 \mathrm{vs} 0 \mathrm{ug} / \mathrm{mL}$ for renal pelvis, $0.65 \mathrm{vs} 0 \mathrm{ug} / \mathrm{mL}$ for renal cortex, $1.34 \mathrm{vs} 0.26 \mathrm{ug} / \mathrm{mL}$ for spleen and 3.36vs1.36 ug/mL for liver, respectively). Conclusions: We posit that the USPIO coated with ALN-PEO is stable, tunable and non-cytotoxic, permitting its further development in multimodal USPIObased theranostics. By targeting activated macrophages, FAPM demonstrate the potential for assessing image-guided drug pharmacokinetics.

Supported by: NIH P01DA028555, R01NS036126, R01NS34239 (HEG)

\section{THE MOTION CONTROL OF FE3O4 NANOMATERIAL AND ITS APPLICATIONS IN NANOMEDICINE}

Liu D, Gabayno JL, Chang M. Chung Yuan Christian University, Chung Li, Taoyuan, Taiwan.

In this paper, we report controlled motion of magnetic nano/micro materials ( $\mathrm{Fe} 3 \mathrm{O} 4)$ which are remotely driven to assemble, rotate, and propel in suspension by oscillating magnetic fields. The propulsion of the nanomaterials is guided directly by an array of permanent magnets and a solenoid connected to an AC source. The magnetic field induction in the immediate vicinity of the sample is controlled by varying the current and frequency of the oscillating field. Optical inspection using a high speed camera tracks the motion of the nano/microstructures.

In the suspension, the nanomaterials assemble into ellipsoidal microstructures. These are magnetized by the permanent magnets to align the magnetic moments along with the field. The oscillating field creates the torque on the microstructures to create rotation about its long axis. A strong static field is also applied to generate a high gradient magnetic field. This gradient produces the thrust to propel the microstructures. The length and diameter of the structures are also controlled by the static field. For structures $10 \mu \mathrm{m}$ in length and 1 micrimeter in diameter, the onset of spiral motion is observed if the 
oscillating field amplitude is 5 gauss. Translation is initiated at 10 gauss and the field gradient is $0.0286 \mathrm{~T} / \mathrm{m}$. At 20 gauss and field gradient of $0.0454 \mathrm{~T} / \mathrm{m}$, the rotational and linear speeds are $47.1 \mathrm{rad} / \mathrm{s}$ and $15.4 \mu \mathrm{m} / \mathrm{s}$, respectively.

From the above results, we demonstrate the capability of the system by translating the microstructures to a target area and switch on the oscillating field to produce rotation. This controlled motion is applied for the fast removal of thrombus. This shows that non-toxic, cost-effective, and batch-produced nanomaterials can be tailored to behave in a controllable fashion as scalable nano/micro motors. Its application for mixing nonmagnetic carrier agents in blood is also being explored.

068. NANOTHERAPEUTIC APPROACH FOR TARGETING THE HIV RESERVOIR IN THE BRAIN Mahajan $\mathrm{SD}^{1}$, Yu Y ${ }^{2}$, Chen $\mathrm{C}^{2}$, Aalinkeel $\mathrm{R}^{1}$, Reynolds $\mathrm{JL}^{1}$, Nair $\mathrm{BB}^{1}$, Sykes $\mathrm{DE}^{1}$, Mamman $\mathrm{M}^{1}$, Cheng $\mathrm{C}^{2}$, Schwartz $\mathrm{SA}^{1}$. ${ }^{1}$ State University of New York at Buffalo, Medicine, Buffalo, NY 14214 and ${ }^{2}$ State University of New York at Buffalo, Chemical and Biological Engineering, Buffalo, NY, 14214.

Background: Nanotechnology can revolutionize the field of HIV medicine by improving delivery of antiretrovirals to targeted regions in the body and by significantly enhancing the efficacy of the currently available antiretroviral medication. Development of a biocompatible nanoformulation that can target HIV-1 in sequestered sites requires the use of functionalized nanoparticles that are engineered to deliver drugs to specific sites in the body where the virus is sequestered, such as the brain. To establish proof of concept, we choose the protease inhibitor "Atazanavir" which is an antiretroviral drug with limited Blood Brain Barrier (BBB) permeability. Methodology: We developed a biodegradable Poly(lactic-co-glycolic acid) - nanoparticles (PLGA-NPs) nanoformulation of Atazanavir and evaluated its ability to effectively cross the BBB and target specific HIV-1 infected immune cells such as monocytes and macrophages and HIV-1 infected neuronal cells in the brain parenchyma. Further, we evaluated the efficacy of this nanoformulation in-vitro using a 3 dimensional dynamic in-vitro BBB model (DIV-BBB). Results: Our confocal imaging results show a significant increase in uptake of the Atazanavir nanoformulation as compared to Atazanavir alone by neuronal and immune cells in-vitro. Additionally, we not only observed a significant increase in the transversing ability of the nanoformulation across the in-vitro BBB as compared to Atazanavir alone, but also observed a significant decrease $(80 \%$; $p<0.01)$ in $\mathrm{p} 24$ levels in HIV -1 infected monocytes treated with the nanoformation as compared to those treated with Atazanavir alone. Conclusion: Our results suggest that the nanoformulation can provide sustained release of the antiretroviral in the CNS, as well as target immune cells such as HIV-1 infected monocytes and macrophages in the brain thereby eliminating the sequestered virus from the brain.

069. NANOART INDUCED BIOLOGICAL RESPONSES IN MURINE EXUDATES

Martinez-Skinner A, McMillan J, Gendelman HE. Department of Pharmacology and Experimental Neuroscience, University of Nebraska Medical Center, Omaha, NE 68198.

Eradication of human immunodeficiency virus (HIV) requires penetrance of long-acting antiretroviral therapy (ART) into viral reservoirs. We embraced this requirement by the development of nanoformulated ART (nanoART). Amongst its unique properties rests the ability to activate monocytemacrophages leading to enhanced cell migratory, phagocytic, and secretory functions. Although nanoART's cellular carrying capacities are known in vitro little has been uncovered in vivo. To this end we assessed cellular temporal uptake of nanoART in murine peritoneal cell exudates following nanoART treatment. Here, balb/c mice were injected by the intraperitoneal route with $100 \mathrm{mg} / \mathrm{kg}$ of nanoatazanavir (nanoATV) and peritoneal exudates collected 4, 12, 24, and $48 \mathrm{~h}$ later. Fluorescence-activated cell sorting facilitated staining for Cd11b, F4/80, and GR-1. Cellular fractions, plasma and lymph nodes were analyzed by high performance liquid chromatography for drug. NanoATV induced equivalent cellular responses as was observed by Zymosan A (inflammatory agent) with the sole exception of the macrophage population. Surprisingly, macrophages appeared 2 logs below Zymosan A treatment after $4 \mathrm{~h}$ of nanoATV treatment. While macrophage cell numbers rose at $12 \mathrm{~h}$, they remained lower than controls without evidence of direct cytotoxicities. NanoATV concentrations among sorted cell populations at each of the time points indicate high specificity of drug particles for macrophages independent of cell numbers. Notably, ATV concentrations within lymph nodes were significant after $4 \mathrm{~h}(4 \mu \mathrm{g} / \mathrm{g}$ tissue) without change after 12, 24, and $48 \mathrm{~h}$. Overall, these data demonstrate the targeting ability of nanoART and the biological effects of particle-cell interactions on cell migration. The results support the idea that macrophages traffic rapidly after exposure to nanoART and to regional lymph nodes. Such data further support the promise of nanoART in its potential to eradicate viral reservoirs.

Supported by: NIH P01DA028555, R01NS036126, R01NS34239 (HEG)

070. ASSESSING POSSIBLE TOXICOLOGICAL EFFECTS OF CARBON NANOPARTICLES ON LIVER AND KIDNEY OF RATS

Mendonça $\mathrm{D}^{1,2}$, Soares $\mathrm{E}^{2}$, Ceraglioli $\mathrm{HJ}^{3}$, Baranauskas $\mathrm{V}^{3}$; da Cruz-Höfling MA ${ }^{1,2} .{ }^{1}$ Department of Pharmacology, Faculty of Medical Sciences, Zip Code 13083-887, Campinas, Brazil, ${ }^{2}$ Department of Histology and Embryology, Institute 
of Biology, State University of Campinas (Unicamp), Zip Code 13083-863, Campinas, Brazil, ${ }^{3}$ Department of Semiconductors, Instruments and Photonics, School of Electrical and Computer Engineering, State University of Campinas (Unicamp).

Carbon nanoparticles, such as Graphene oxide (GO), have emerged as instrumental for biomedical applications and nanomedicine because of their unique physical, chemical, electrical, and mechanical properties. In recent years, conflicting data have been reported about possible toxicological implications of carbon nanoparticles in nanomedicine. Herein, we investigate whether GO nanoparticles dispersed in $\mathrm{H} 2 \mathrm{O}$ and GO dispersed in polyethylene glycol (PEG) $(1 \mathrm{mg} / \mathrm{ml})$ affects the indicators of kidney (urea and creatinine) and liver (alanine transaminase (ALT) and aspartate transaminase (AST)) health. GO nanoparticles were administered to 6week-old male Wistar rats, (Rattus novergicus) at a dose of $7 \mathrm{mg} / \mathrm{kg}$ by intravenous injection into the tail vein $(n=3 /$ group) and kidney and liver parameters examined at different time intervals ( $15 \mathrm{~min}, 1 \mathrm{~h}, 3 \mathrm{~h}$ and 7 days). Statistical analysis was performed using unpaired Student's $t$-test; data were expressed as mean \pm SEM with $p \leq 0.05$ indicating significance when compared to the corresponding control. The experiment followed COBEA guidelines and was approved by the University's Committee for Experimental Use of Animals (CEUA, protocol 2884-1). The treatment with GO-H2O caused only an $18 \%$ increase of creatinine at $15 \mathrm{~min}$ $(p \leq 0.05)$, whereas the hepatic parameters remained normal. Moreover, the serum level of urea decreased transiently by $40 \%$ at $3 \mathrm{~h}(p \leq 0.01)$ with GO-H2O and the serum levels of hepatic AST decreased by $64 \%$ after 7 days treatment with GO-PEG $(p \leq 0.05)$ indicating improvement of kidney and hepatic functional parameters. No histopathological changes were detected in groups. The data demonstrated that carbon nanoparticles exposure to rats present minimal toxicological effect at the experimental conditions used.

\section{LIPID-BASED VACCINE NANOPARTICLES FOR INDUCTION OF ROBUST CELLULAR AND HU- MORAL IMMUNE RESPONSES AGAINST MALARIA AND HIVANTIGENS}

Moon JJ. University of Michigan, Ann Arbor, MI 48109.

There is a great interest in new vaccine technologies that can elicit robust cellular and humoral immune responses. We have developed a novel vaccine platform, called interbilayercrosslinked multilamellar vesicles (ICMVs), and demonstrated their efficacy to elicit immune responses against subunit antigens. ICMVs were formed by fusing liposomes into multilamellar vesicles and subsequent crosslinking of adjacent lipid headgroups across lipid bilayers within multilamellar vesicles (Moon et al. Nat Mat 2011). These nanoparticles exhibited substantially enhanced protein loading and extended drug release kinetics compared with traditional drug delivery vehicles (e.g. liposomes and polymeric particles). Crosslinking of lipid bilayers enhanced stability of particles in serum, allowing efficient delivery of antigens to antigen-presenting cells. ICMVs encapsulating ovalbumin and FDA-approved adjuvant elicited potent antibody and $\mathrm{CD} 8+\mathrm{T}$ cell responses in vivo, comparable to those induced by viral vector vaccines, but without safety issues and anti-vector immunity associated with viral vectors. Furthermore, ICMVs carrying a malaria antigen elicited robust humoral immune responses with high-avidity antibody titers lasting more than a year (Moon et al. PNAS 2012). Lastly, we examined ICMVs as a vaccine carrier for HIV antigens ( $\mathrm{Li}$, Moon et al., Sci Transl Med 2013). Mice were immunized via pulmonary route with a gag HIV peptide in ICMVs had significantly higher frequency of AL-11-specific CD8+ $\mathrm{T}$ cells in lungs, lymph nodes, spleen, gut, and reproductive tract, compared with mice immunized with soluble antigens. These results suggest that ICMVs are a potent vaccine platform that can stably deliver antigen to lymphoid tissues and elicit significantly enhanced cellular and humoral immune responses to subunit antigens.

\section{A NOVEL NANOBEACON FOR IMAGING CO- LORECTAL CANCER}

Pham W. Vanderbilt University School of Medicine, Nashville, TN 37232.

Purpose: The objective of this research is to integrate nanotechnology with molecular imaging for early detection of colorectal cancer (CRC). CRC undergoes a protracted asymptomatic stage before it reaches the advance stage. Therefore, detection in the early onset through regular screening will improve therapeutic outcomes and save lives. In that regard, colonoscopy is considered the golden standard for early detection. However, its effectiveness for early detection of tumor growth is mitigated by its incapacity to disclose molecular level changes. One molecule associated with the development and progress of CRC is the Thomsen-Friedenreich (TF) antigen (Ag). High expression of TF disaccharide in CRC and its absence from normal tissue represents a unique association in CRC that exhibits the qualities of a prognostic biomarker. Method: We developed a fluorescence (FL) nanobeacon, which can bind specifically to TF-associated CRC due the presence of multiple identical copies of peanut agglutinin (PNA) molecules derivatized on the surface of the nanobeacon. The physical property of the FL nanobeacon such as size, shape, surface topography, elemental composition and polymer distribution was evaluated using DLS, SEM, XPS and permeation chromatography, respectively. We assessed the sensitivity 
and specificity of the nanobeacon on human specimens. In addition, we developed an orthotopic rat model of $\mathrm{CRC}$ to assess the specificity of the nanobeacon using white light and FL colonoscopy. Results: The overall size of the nanobeacon is approximately $350 \mathrm{~nm}$. There are approximately 200-300 PNA molecules, which serve as molecular recognition moieties. In vivo imaging data demonstrated that the FL colonoscopy using the nanobeacon detects CRC much earlier than conventional colonoscopy. Conclusion: We demonstrated the potential use of a novel nanobeacon for early detection of CRC. The data suggests that TF Ag can be used as a potential biomarker for imaging CRC.

\section{MACROPHAGE-TARGETED LONG-ACTING} NANOFORMULATED ANTIRETROVIRALTHERAPY

Puligujja P, McMillan J, Balkundi S, Kendrick L, Hilaire J, Bade A, Gorantla S, Poluektova L, Liu X, Gendelman HE. Department of Pharmacology and Experimental Neuroscience, University of Nebraska Medical Center, Omaha, NE 68198.

Background: Patient antiretroviral therapy (ART) adherence, distribution to viral sanctuaries, antiviral responses and optimal pharmacokinetic and pharmacodynamics (PK and PD) underlie the success of drug treatment strategies. To such ends long acting nanoformulated ART (nanoART) was developed. However, progress has been hampered by high dose requirements. We posit that the addition of specific cell targeting ligands, such as folic acid, on the particle surface will improve treatment outcomes. Methods: Folic acid-poloxamer 407 (FA-P407) ritonavir boosted atazanavir (ATV/r) nanoformulations were prepared by high-pressure homogenization and physicochemical parameters determined. ATV/r nanoART cell uptake, drug retention, release and antiretroviral activities were determined in human monocyte-derived macrophages (MDM). PK and PD were performed in Balb/cJ mice after intramuscular (IM) injections. Dose-dependent antiretroviral activities were performed in human peripheral blood lymphocyte reconstituted NOD/scid-IL2Rgc (NSG huPBL) mice-infected with HIV-1ADA. Immune profiles (CD4+/CD8+ T cell ratios); HIV-1p24 RNA and antigen were determined in infected spleens. Results: FA coated nanoART demonstrated $>2$-fold higher drug uptake, retention and release in MDM than untargeted formulations. In Balb/cJ mice, plasma ATV levels were $400 \mathrm{ng} / \mathrm{ml}$ and 5-fold higher than uncoated formulations. Tissue drug levels (liver, kidney, spleen and lymph) increased from 2-5-fold by FA coatings. Antiretroviral activities were dose dependent, with little or no detectable HIV-1 p24 antigen and HIV-1p24 RNA 14 days following a single IM injection with 50 and $100 \mathrm{mg} / \mathrm{kg}$ doses. Folate targeting facilitated drug depots in recycling endosomes. Serum chemistry tests showed normal metabolic profiles. Conclusions: These data, taken together, show superior PK and PD and antiretroviral responses in targeted nanoART formulations demonstrating potential for human use.

Supported by: NIH P01DA028555, R01NS036126, R01NS34239 (HEG)

\section{PROTON BEAM TRIGGERED NANOPARTICLE DRUG DELIVERY FOR TARGETED CONCURRENT CHEMORADIOTHERAPY}

Ranjan A and Fernando R. Oklahoma State University, Stillwater, OK 74074 .

Purpose: The objectives of this study were to: 1) determine if mild hyperthermia $\left(40-42{ }^{\circ} \mathrm{C}\right)$ can sensitize tumor cells for more effective proton beam radiotherapy (PBRT); 2) characterize the survival fraction of cells exposed to PBRT; and 3) characterize release of the drug doxorubicin (Dox) from low temperature sensitive liposomes (LTSLs) without exposure to mild hyperthermia in combination with PBRT. Methods: Dox was actively loaded in LTSLs. A549 monolayer cells were incubated with 100-200 nM of Dox-LTSL ( \pm mild hyperthermia). Cell irradiation ( $0-6 \mathrm{~Gy}$ ) was performed by placing the cell culture plates inside a solid water phantom and using a clinical proton treatment beam with energy of $150 \mathrm{MeV}$. End points were survival fraction, radiation-mediated Dox release, and reactive oxygen species (ROS) production. Results: Hyperthermia effectively sensitized cells for PBRT and lowered the cell survival fraction (SF) by an average of $9.5 \%$. The combination of $100 \mathrm{nM}$ Dox-LTSL and PBRT (1-6 Gy) achieved additive to synergistic response at various dose combinations. At higher radiation doses ( $>3 \mathrm{~Gy}$ ), the SF in the Dox and DoxLTSL groups was similar $(\sim 20 \%)$, even in the absence of hyperthermia. In addition, $30 \%$ of the Dox was released from LTSLs and a 1.5-2 fold increase in ROS level occurred compared to LTSL alone therapy. Conclusion: The combination of LTSLs and PBRT can achieves additive to synergistic effect at various dose combinations in vitro. Concurrent PBRT and DoxLTSL treatment significantly improved the cytotoxic outcomes of the treatment compared to PBRT and Dox chemotherapy without LTSLs. We hypothesize that PBRT may induce drug delivery from LTSL in the absence of hyperthermia.

\section{ROLE OF CARRIER SIZE ON THE TRANSPORT OF DRUG CARRIERS ACROSS CELLULAR BARRIERS}

Serrano $D^{1}$, Chadha $\mathrm{R}^{2}$, Muro $\mathrm{S}^{2,3} .{ }^{1}$ Biological Sciences Graduate Program, ${ }^{2}$ Institute for Bioscience and Biotechnology Research, and ${ }^{3}$ Fischell Department of Bioengineering, University of Maryland, College Park, MD 20742.

Transport of drug carriers across cell layers (e.g. for delivery across the blood-brain barrier) remains a major challenge. Targeting cell surface receptors involved in vesicular transport via transcytosis across these linings has shown promise to 
overcome this obstacle. However, how carrier biophysical parameters affect transcytosis remains unexplored. Since carrier size impacts rate and mechanism of uptake by cells, we focused on this parameter. As an example, we examined ICAM-1targeted carriers. ICAM-1 is a transmembrane protein involved leukocyte transit from blood into tissues, and supports transport of drug carriers across cells via cell adhesion molecule (CAM)mediated endocytosis. CAM-uptake differs from clathrin- and caveolae-mediated endocytosis, allowing for uptake of carriers of a wide range of sizes (100 $\mathrm{nm}$ to several $\mu \mathrm{m}$ in diameter). This flexibility in uptake makes the CAM-pathway suitable to study the role of carrier size in transcytosis. We used model polymer carriers of different sizes coated with antibodies to ICAM-1 to study transport into and across endothelial monolayers. We found that, although increasing carrier size decreased the number of carriers bound to cells (from 57.3 to 2.2), the cellsurface area occupied by bound carriers increased with carrier size ( 0.45 to $\left.34.6 \mu \mathrm{m}^{\wedge} 2\right)$. Despite this, efficiency of endocytosis (\% carriers internalized) was high and independent of carrier size (90 to $95 \%$ ). As per intracellular distribution, increasing carrier size lowered transport to lysosomes (from 85 to $40 \%$ lysosome colocalization). Surprisingly, yet in agreement with lower lysosomal transport, increasing carrier size augmented the efficacy of transport across cells, with micro-carriers exhibiting higher efficiency of transport than nano-carriers (37.6 vs. 14.2\% transport). These results illustrate the role of carrier size in modulating transport across cell layers and hold potential to guide drug delivery across cell linings, e.g. into the brain.

076. SYNTHESIS, CHARACTERIZATION AND EVALUATION OFA CORE-SHELL NANOCOMPOSITE AS ORALLY INGESTIBLE MAGNETIC RESONANCE CONTRAST AGENT

Shanavas $\mathrm{A}^{1}$, Bahadur $\mathrm{D}^{2}$, Srivastava $\mathrm{R}^{1} .{ }^{1} \mathrm{BSBE},{ }^{2} \mathrm{MEMS}$, Indian Institute of Technology Bombay, Mumbai, India.

Iron oxide nanoparticles (IONP) are T2 Magnetic Resonance (MR) contrast agents, when taken orally are limited to gastrointestinal (GI) imaging [1]. While the available reports warrant them as safe contrast agents via intravenous route, at least one report has shown $8 \%$ of patients having fatal anaphylactic reactions after receiving a bolus injection [2]. The current work explores possibility of delivering IONPs across the intestinal barrier to circulating blood to be targeted to a distant diseased tissue especially hepatic lesion. Tackling the intestinal mucosal barrier for effective delivery to systemic circulation is the challenge, as the carrier of IONPs should clearly adhere onto the intestinal epithelium to follow an uptake pathway. A submicron (200 $\mathrm{nm}$ or less) size and cationic to near neutral particle surface charge are important criteria for increased residence time, as glycocalyx making the mucosa is negatively charged. We are reporting a Folate-chitosan coated IONP-PLGA nanoparticles having a core-shell nanostructure, where shell protects the core containing IONPs from degradation, stable at varying GI $\mathrm{pH}$ and enhances GI uptake of the particles. The material has been characterized for Size $(<200 \mathrm{~nm})$, Charge (positive to near neutral), IONP encapsulation efficiency $(60 \%)$, folate chitosan coating efficiency (via Nitrogen-Carbon ratio) and IONP relaxivity after loading/ coating $(60 \mathrm{mM}-1 \mathrm{~s}-1)$. The material was subjected to phantom MR imaging of HepG2 hepatocellular carcinoma cells, which showed enhanced contrast due to their cationic nature. As these particles were found to enhance MR contrast of oral cancer cells, KB, via folate receptor targeting, future biodistribution studies may pour insights if folic acid can facilitate targeting these structures to any non-hepatic tissues, after GI absorption.

\section{NANOBIOSENSING DEVICES FOR DETECTION OF MESOTHELIN IN CANCER CELL LINES}

Singh $\mathrm{KP}^{1}$, Puri $\mathrm{A}^{2}$, Ahlawat $\mathrm{S}^{1}$, Prusty AK ${ }^{1}$. ${ }^{1}$ Bio-Nanotechnology Research Laboratory, Biophysics Unit, G.B. Pant University of Agriculture and Technology, India, ${ }^{2} \mathrm{Basic}$ Research Lab, Center for Cancer Research, National Cancer Institute, Frederick, MD 21702-1201.

Metallic, polymeric and carbon-based materials and recently graphene-based materials are considered viable choices for signal amplification of biosensors. We have proposed a flow injection assay of the mesothelin using quartz crystal microbalance (QCM) biosensor. Gold-coated quartz crystal functionalized with 16-mercaptohexadecanoic acid (MHDA) self assembled monolayer was used to fabricate a sensitive immunobiosensor for mesothelin detection and compared with gold nanoparticles (AuNPs) and graphene oxide (GONPs) nanoparticles. Mesothelin specific polyclonal antibody was covalently immobilized on MHDA modified gold surface of quartz crystal using N-ethyl-N'-(3-dimethylaminopropyl) carbodiimide and N-hydroxysuccinimide (EDC/NHS) chemistry. Ethanolamine was used as a blocker to prevent nonspecific adsorption. A homemade siphon flow system was designed using a clamp stand, Stanford Research System-flow cell, precision silicon tubing, stopper, 6-port valve and syringe. Phosphate buffer saline solution of $\mathrm{pH} 7.4$ was used as the carrier buffer@ rate $40 \mu$ liters per minute for the experiment to get the stable base line of the resonance frequency of the crystal. Different concentrations of pure mesothelin, AuNPmesothelin and GONP-mesothelin were allowed to flow for binding with the antibody immobilized on the surface modified crystals. The resonance frequency variation of the crystal was observed by a quartz crystal microbalance until a stable response is obtained for various concentrations of mesothelin. The frequency changes revealed a close correlation with Mesothelin concentrations in the measurement range of 100 pg.mL-1 to 100 ng.mL-1. The GO-coated sensing units provide improved sensitivity of mesothelin detection. 
Supported by: This project is part of the joint venture supported by Intramural to India (I-to-I) funding for treatment and prevention of sexually transmitted diseases awarded to Dr. Anu Puri (NCI) and Dr. K.P. Singh (Pantnagar University).

\section{STUDIES ON ENCAPSULATION OF PHOTO- DYNAMIC ANTICANCER AGENTS, CHLORIN E6 AND HPPH IN PHOTOACTIVABLE "POCKET" LIPOSOMES CONTAINING DC8,9PC}

Thayer D, Sine J, Blumenthal R, Puri A. Basic Research Lab, Center for Cancer Research, National Cancer InstituteFrederick, Frederick, MD, 21702.

We recently reported the release of a water soluble dye, calcein (Ex/Em 490/517 nm), from liposomes containing dipalmitoyl phosphatidylcholine (DPPC) and DC8,9PC upon laser treatment. DC8,9PC has the hypothesized effect of forming pockets in the liposome membrane allowing for photo-activated compounds to more easily permeate the liposomal membrane. To develop these formulations for dual drug delivery and in vivo applications, we have utilized two near-infrared Photodynamic Therapy (PDT) drugs, Chlorin e6 (ex/em 400/660 nm) and 2-(1-Hexyloxyethyl)2-devinyl pyropheophorbide-a (HPPH) (ex/em 410/670 nm). Liposomes loaded with calcein and with or without either of the PDT drugs underwent $660 \mathrm{~nm}$ laser-triggered release of calcein and were analyzed for fluorescence. Our data shows that inclusion of HPPH in the formulations enhanced calcein entrapment. In contrast, Chlorin e6 had significant reduction in the entrapment of calcein under identical conditions. Control samples (loaded only with calcein) did not promote calcein release upon $660 \mathrm{~nm}$ laser exposure. Based on our observations, we have reason to believe that the two PDT drugs partition into the lipid membrane through different mechanisms. These differing mechanisms affect how calcein is encapsulated into the aqueous core of the liposome. Therefore, we propose that HPPH is preferential to Chlorin e6 in our phototriggerable drug delivery platforms, while the specific dynamics of membrane partitioning still remain unknown. Going forward, the efforts of this research will be to determine the specific partitioning mechanism of HPPH as well as conclude the optimum procedure for dualdrug delivery through our liposome formulations.

\section{ADVANCED EFFICIENCYAND SPECIFICITY OF} ANTI-HIV DRUGS CONJUGATED WITH NANOGELS Warren $\mathrm{G}^{1}, \mathrm{Lu} \mathrm{Y}^{1}$, Makarov $\mathrm{E}^{2}$, Senanayake $\mathrm{T}^{1}$, Gorantla $\mathrm{S}^{2}$, Poluektova $\mathrm{LY}^{2}$, Vinogradov SV ${ }^{1}$. ${ }^{1}$ Department of Pharmaceutical Sciences and ${ }^{2}$ Department of Pharmacology and Experimental Neuroscience, University of Nebraska Medical Center, Omaha, NE 68198.

The effectiveness of anti-HIV drugs is limited to their ability to accumulate in infected immune cells. Here we introduce novel targeted nanogel conjugates with nucleoside reverse transcriptase inhibitors (NRTIs) with enhanced antiviral activity, which are able to efficiently accumulate in macrophages. In the first type of conjugates, NRTIs are connected to nanogels via biodegradable succinate linker, while in the second type NRTIs are attached via an oligophosphate linker. Nanogel design was based on nontoxic biodegradable biopolymers (e-polylysine and dextrin) modified by cholesterol moieties and formed compact particles with hydrophobic core after sonication in aqueous media. High drug loading (up to $40 \%$ by weight) could be obtained in these nanogel-NRTIs. Nanogel-linked Zidovudine, Lamivudine or Abacavir, components of therapeutic Combivir and Trizivir cocktails, have been evaluated in HIV-infected human monocyte-derived macrophage (MDM). Ten-fold inhibition of HIV-1 activity in the infected MDMs was achieved at concentration two to nine times lower compared to conventional drugs. Dual- and triple-drug nanogel-NRTI cocktails, similar to therapeutic NRTI cocktails, also showed significantly higher antiviral activity in the infected MDMs compared to nanogel-NRTIs. Novel nanodrugs allowed an extended, up to 10 days, drug release kinetics, and exhibited preferential uptake in macrophages. Efficacy of nanogel-NRTI uptake was enhanced threefold by the covalent modification with glutathione moieties. In addition, these antivirals demonstrated much lower toxicity in variety of cells (e.g., macrophages, brain capillary endothelial cells, astrocytes, microglia, etc.) than NRTIs. Additional modification with brain-specific peptides was successfully applied to the CNS delivery of nanogel-NRTIs. Current work will be extended for evaluation of antiviral efficacy using an in vivo humanized HIV mouse model in order to assess therapeutic advantages of new nanodrugs.

080. NOVEL SELF-ASSEMBLY AND PHSENSITIVE ANTICANCER DRUG CARRIERS (POLY(AMIDO AMINE)-ANILINE PENTAMER)MODIFIED POLYETHYLENE GLYCOL LOADINGWITH DOXORUBIC

Yeh J-M, Chang, K-C, Wu P-S. Chung Yuan Christian University Taiwan 32023, R.O.C.

A novel system that self-assembly and $\mathrm{pH}$-sensitive drug release is based on aniline pentamer (AP) as a shell conjugated to polyethylene glycol (PEG)-modified dendrimers (PAMAM) with doxorubicin (DOX) (DOX-PEG-PAMAMAP) has been constructed and characterized. Firstly, the higher generations $(\mathrm{G}=5)$ are demonstrated to self-assemble into bilayer vesicles owing to the strong tendency of the AP to form PAMAM-AP G5. Subsequently, PAMAM-AP G5 modified hydrophilic and biocompatibility segment PEG synthesis of PEG-PAMAM-AP G5. Then, load with the anticancer drug (DOX) to synthesis DOX-PEG-PAMAM-AP G5. The use of amphiphilic characteristics form these aggregations as new drug carrier to explore the ability to cytotoxicity of human breast cancer cells (MCF-7), and compare the drug release 
efficiency. The results show that the novel anticancer drug carriers (DOX-PEG-PAMAM-AP G5) could effectively enhance the DLE and drug cumulative release at $\mathrm{pH}=4.0 \mathrm{com}$ pared with DOX-PEG-PAMAM G5. The cytotoxicity of MCF-7 of DOX-PEG-PAMAM-AP G5 is similar to DOX.

\section{OPTICALLY-ENHANCED DELIVERY AND TRACKING OF THERANOSTIC NANOPARTICLES IN GLIOBLASTOMA IN ANIMAL MODEL}

Yuan H. Duke University, Durham, NC 27708.

Cancer nanotheranostics provide tremendous biomedical opportunities for cancer diagnosis, imaging, and therapy. Such a "theranostics" strategy could potentiate translational research and improves precision medicine. Brain malignancy, due to the presence of blood-brain barrier (BBB) that hinders paracellular flux of drug molecules into brain parenchyma, remains one of the most challenging tumor types. Great difficulty existed in designing a theranostic agent with enhanced property to enter brain tumor parenchyma. In this article, we present a theranostic nanoplatform that acts as contrast agent, photothermal therapeutic agent, and blood-brain tumor barrier (BBTB) permeabilization agent. We first demonstrate a superior optical property of our nanoplatform as contrast agent under high-resolution depth-resolved multiphoton microscopy. We then examine the microscopic distribution of PEGylated nanoparticles in tumor brain animal models. Nanoparticles were found in both endothelial cells and interstitial matrices. We also examine the effect of different diameters and two potential BBTB penetrating surface functionalizations (e.g., TAT peptide, angiopep2 peptides). Furthermore, since current BBB disrupting agents (e.g., mannitol, focused ultrasound) typically suffer from poor spatial control, we demonstrated a novel photothermal-induced BBTB permeabilization with highly selective spatial delivery. Fine-tuning the irradiating energy induced gentle disruption of the vascular integrity and secondary inflammatory reaction, causing short-term extravasation of nanoparticles into tumor parenchyma only at the irradiated site. Based on these findings, we conclude that our theranostic nanoplatform is both a strong biocompatible contrast agent and a powerful BBTB permeabilizing agent. With more optimization, we envision a strong potential in future brain tumor imaging and therapy.

082. A MIXED-LINEAGE KINASE 3 INHIBITOR FACILITATES NANOFORMULATED ANTIRETROVIRAL THERAPY CLEARANCE OF HIV-1 ASSEMBLY Zhang $\mathrm{G}^{1}$, Dash $\mathrm{PK}^{1}$, Guo $\mathrm{D}^{1}$, Wiederin $\mathrm{JL}^{1}$, Ciborowski $\mathrm{P}^{1}$, Goodfellow $\mathrm{VS}^{2}$, McMillan $\mathrm{J}^{1}$, Gorantla $\mathrm{S}^{1}$, Gelbard $\mathrm{HA}^{3}$, Gendelman $\mathrm{HE}^{1}{ }^{1}{ }^{1}$ University of Nebraska Medical Center, Omaha, NE, 68198; ${ }^{2}$ Califia Bio, Inc., San Diego, CA, 92121; ${ }^{3}$ University of Rochester Medical Center, Rochester, NY, 14642.
Antiretroviral therapy (ART) has improved the quality of life for infected people. However, despite ART viral replication continues, albeit at low levels in reservoirs of persistent infection. Eradication of infection remains the major goal of HIV researchers. Recently, a novel mixed-lineage kinase-3 (MLK3) inhibitor (URMC-099) with promising immune and neural modulatory activities was discovered. Surprisingly, the drug potentiated antiretroviral activities of nanoformulated long-acting ART. Isobaric tag for relative and absolute quantitation (iTRAQ) performed using the mixed-lineage kinase URMC-099-treated macrophages potentiated antiretroviral actions. Nanoformulated atazanavir (nanoATV) with dose escalated URMC-099 was tested for antiretroviral activities in HIV-1ADA infected MDM. Viral DNA and RNA, Gag proteins and reverse transcriptase activities were assessed. Subcellular endosomal trafficking (Rab 5, 7, 8 and 14) of ART nanoparticles were investigated by Western blot, confocal microscopy and HPLC. Humanized NOD/scid-IL2Rgcnull mice were infected with HIV-1ADA then URMC099 and nanoATV/r. Peripheral viral load (VL), human CD4+ T cells, and lymphoid and brain tissues were studied. URMC-099 increased expression of the RAS-related GTP-binding proteins Rab 7, 8 and 14 in HIV-1 infected MDM. NanoATV antiretroviral activities were potentiated by URMC-099 with reductions in viral gene products. URMC-099 increased ATV concentrations in Rab7 endosomes. Highest CD4+ T cell numbers and lowest VL were in infected humanized mice treated with URMC-099 and nanoATV. Histopathological tests showed decreased numbers of HIV-1p24 cells with both drugs compared to nanoATV alone in lymphoid tissues. iTRAQ showed Rab7 changes specifically in infected MDM. URMC-099 potentiates nanoATV responses to restrict HIV-1 and protect CD4+ T lymphocytes. This occurs, in part, by sustaining ART levels. These effects may represent a novel means for viral clearance. Supported by: NIH P01DA028555 and R01 A1097550 (HEG); P01MH064570 (HAG)

\section{TARGETED (-)-EPIGALLOCATECHIN GAL- LATE LOADED LIPID NANOPARTICLES INHIBIT FOAM CELL FORMATION}

Zhang J, Nie S, Wang S. Nutritional Sciences Program, Texas Tech University, Lubbock, TX 79409.

Macrophages play an important role in atherosclerotic lesion progression by facilitating cholesterol accumulation and increasing inflammatory responses in aortic walls. After accumulating cholesterol, macrophages can be transformed into foam cells, which characterize the atherosclerotic lesion. (-)Epigallocatechin gallate (EGCG), a natural compound found in green tea, has a potential to inhibit foam cells formation through decreasing macrophages cholesterol accumulation and inflammatory response. We have successfully synthesized targeted EGCG loaded lipid nanoparticles (T-nanoE). The 
EGCG stability was significantly increased by the nanoparticles. T-nanoE had higher binding affinity to macrophages than non-targeted EGCG loaded lipid nanoparticles (nanoE). After knocking down a scavenger receptor, T-nanoE had a low binding affinity to macrophages. As compared to native EGCG, EGCG nanoparticles significantly decreased macrophage cholesteryl ester content measured using a high perfor- mance liquid chromatography (HPLC) system. EGCG nanoparticles also decreased the expression of monocyte chemoattractant protein 1 (MCP-1) in macrophages. In conclusion, lipid nanoparticles increase EGCG stability. T-nanoE can target to macrophages. Both T-nanoE and nanoE inhibit foam cell formation. T-nanoE might be better than nanoE. Supported by: NIH NCCAM Grant Number R15AT007013 